\title{
TU/e EmonONEN

\section{Numerical simulation of viscous sintering by a periodic lattice of a representative unit cell}

\section{Citation for published version (APA):}

Vorst, van de, G. A. L. (1995). Numerical simulation of viscous sintering by a periodic lattice of a representative unit cell. (RANA : reports on applied and numerical analysis; Vol. 9508). Technische Universiteit Eindhoven.

\section{Document status and date:}

Published: 01/01/1995

\section{Document Version:}

Publisher's PDF, also known as Version of Record (includes final page, issue and volume numbers)

\section{Please check the document version of this publication:}

- A submitted manuscript is the version of the article upon submission and before peer-review. There can be important differences between the submitted version and the official published version of record. People interested in the research are advised to contact the author for the final version of the publication, or visit the $\mathrm{DOI}$ to the publisher's website.

- The final author version and the galley proof are versions of the publication after peer review.

- The final published version features the final layout of the paper including the volume, issue and page numbers.

Link to publication

\section{General rights}

Copyright and moral rights for the publications made accessible in the public portal are retained by the authors and/or other copyright owners and it is a condition of accessing publications that users recognise and abide by the legal requirements associated with these rights.

- Users may download and print one copy of any publication from the public portal for the purpose of private study or research.

- You may not further distribute the material or use it for any profit-making activity or commercial gain

- You may freely distribute the URL identifying the publication in the public portal.

If the publication is distributed under the terms of Article 25fa of the Dutch Copyright Act, indicated by the "Taverne" license above, please follow below link for the End User Agreement:

www.tue.nl/taverne

Take down policy

If you believe that this document breaches copyright please contact us at:

openaccess@tue.nl

providing details and we will investigate your claim. 
EINDHOVEN UNIVERSITY OF TECHNOLOGY

Department of Mathematics and Computing Science

\author{
RANA 95-08 \\ June 1995
}

Numerical Simulation of Viscous Sintering

by a Periodic Lattice of a Representative

Unit Cell

by

G.A.I. van de Vorst

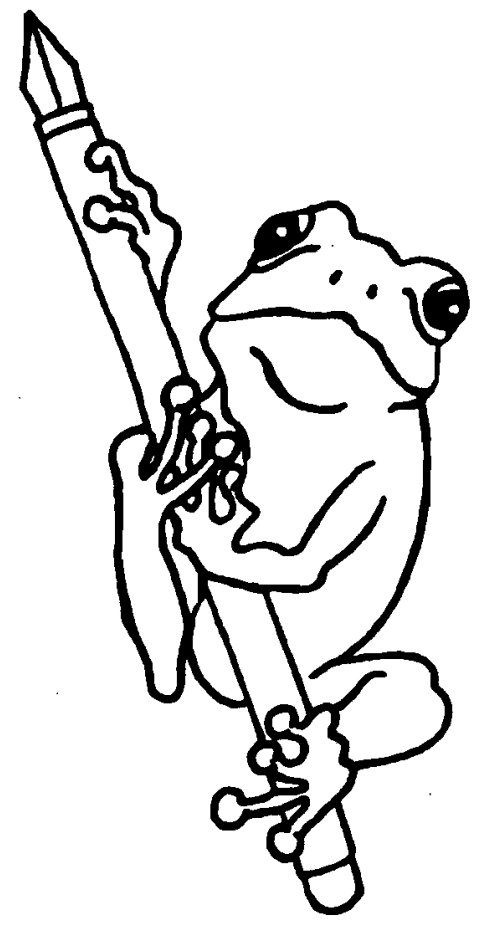


Reports on Applied and Numerical Analysis

Department of Mathematics and Computing Science Eindhoven University of Technology

P.O. Box 513

5600 MB Eindhoven

The Netherlands

ISSN: 0926-4507 


\title{
Numerical Simulation of Viscous Sintering by a Periodic Lattice of a Representative Unit Cell
}

\author{
G.A.L. van de Vorst \\ Department of Mathematics and Computing Science \\ Eindhoven University of Technology \\ P.O. Box 513, $5600 \mathrm{MB}$ Eindhoven, The Netherlands \\ (e-mail:wsangv@win.tue.nl)
}

\begin{abstract}
In this paper numerical simulations of the viscous sintering phenomenon are presented, i.e. of the process that occurs (for example) during the densification of a porous glass heated to such a high temperature that it becomes a viscous fluid. The numerical approach consists of simulating the shrinkage of a two-dimensional unit cell which is in some sense representative for the porous glass. Hence it is assumed that the microstructure of the glass can be described by a periodic continuation in two directions of this unit cell. In this way it is possible to obtain quite a few theoretical insights of the viscous sintering process with respect to both pore size and pore distribution of the material. In particular this model is able to examine the consequences of microstructures on the evolution of the pore size distribution. It appears that only for higher densities the numerical densification rate of one cylindrical pore in a square unit cell is in agreement with the widely used closed pores model of Mackenzie and Shuttleworth. However, the major finding is that the pores vanish in order of size one after another; so the smallest pores first, followed by the larger ones. Moreover it is shown that pores with concave boundary parts may initially grow before they start shrinking at a later time stage.
\end{abstract}




\section{Introduction}

In the last decade there has been a renewed interest in studying the viscous sintering process. This type of process occurs during the sintering of amorphous porous materials such as glass. The interest is mainly due to chemical processing techniques to produce high performance advanced materials. The use of so-called sol-gel techniques for preparing glasses and crystalline ceramics offers an outstanding opportunity to produce such materials.

In sol-gel processing, colloidal particles in a suspension, called a sol, is heated or mixed with a liquid (acid or basic). This causes the particles to aggregate or polymerize and form a giant aggregate or molecule that extends completely throughout the sol; the substance is said to be a gel. Depending on the way the gel is dried one obtains an aerogel or a xerogel. The fundamental importance of this technique is the ability to tailor the microstructure on molecular length scales. For example, a wide range of gel structures can be obtained by varying the $\mathrm{pH}$ of the liquid. Sol-gel processing can be applied in various fields which includes powder forming, monoliths, fibres and thin film coatings (cf. Brinker and Scherer [1]).

At sintering temperature the dried gel appears to behave like an incompressible Newtonian viscous fluid reasonably well: the Stokes creeping flow equations hold (cf. Kuiken [2]). The viscous volume flow causes the densification of the material. The driving force for this flow is the excess of free surface energy of the porous glass compared to a same quantity of a fully dense glass. Ideally, one wants to produce a dense and homogeneous glass, free from voids and impurities this way. Therefore, one needs a good theoretical understanding of the densification kinetics of the porous glass, i.e. the viscous sintering phenomenon.

In particular, one is interested in the shrinkage rate of the gel as a function of the viscosity and particle size, which reflects how time, temperature and microstructure influence the development of the densification process. Another question is which kind of structural configuration leads to a higher densification rate. Overviewing the literature, the picture emerges that over the years a great deal of experimental evidence has been gathered by sintering scientists, but also that it is apparently very difficult to produce sophisticated mathematical models which have some predictive power.

Clearly, a deterministic description of the viscous flow of a structure as complicated as a dried gel (xerogel or aerogel) is out of the question, even when restricting to a simple Newtonian constitutive model, viz. the Stokes equations. The structure is simply too stochastic for such an attempt to be successful. Hence, the first attempts describing sintering phenomena dealt with the behavior of simple systems only, like the coalescing of two spheres (cylinders), or the sintering of a sphere (cylinder) onto a flat surface, hoping to recover the phenomenological behavior for macroscopic systems by these so-called unit problems. In sintering literature these models are also used, with varying degrees of success, as a diagnostic tool to decide which driving force is responsible for a particular sintering process.

This approach goes back to 1945, when Frenkel [3] described the early stage of the coalescence of two equal spherical particles. He introduced the empirical rule, which is used in most mathematical models of viscous sintering to date, that the work done by surface tension in decreasing the total surface area is equal to the total energy produced by dissipation of the flow. So in applying this energy balance one has to know beforehand both the shape deformation 
and the description of the governing flow field during sintering. However, this shape and flow field evolution are unknown and therefore he used approximate descriptions which only allow to predict rough estimates of the coalescence rate of the spheres. In Van de Vorst [4] it is shown that the famous and widely used Frenkel rule, viz. the width of the neck growing between the two spheres is proportional to the square root of the time, does not fit at all with the numerical solution. This guessing of the geometrical deformation and/or flow field is applied in most of the theoretical studies, cf. Kingery and Berg [5], Exner and Petzow [6], Mackenzie and Shuttleworth [7] and Scherer [8]-[10].

Starting from the surface curvature driven Stokes's flow equations, without any assumption on the shape deformation and flow field, Hopper [11]-[14] solved two simple viscous sintering problems analytically, viz. the coalescence of two equal cylinders (Hopper [11],[12]) and the coalescence of a cylinder on a half-space (Hopper [14]). He applied a conformal mapping technique to solve the Stokes equations in which the time evolution of the shape was described in terms of an equation of motion involving the mapping function. In Hopper [12], the method is demonstrated for a number of regions which are bounded by a simple smooth closed curve. Moreover, Hopper was able to solve some problems for semi-infinite regions also. However, the examples in the referred papers show that his method depends on guessing a mapping function which, initially, has a built-in behavior that describes the time evolution of the shape. The difficulty of finding such a mapping is illustrated in Hopper [13]: the only doubly connected domain he could solve this way was a circular disk with a circular hole, centered at the origin. This problem can also be solved easily with the use of cylindrical coordinates. Recently, Richardson [15] was able to solve the unit problem of the coalescence of two unequal cylinders by applying Hopper's method.

Application of computational methods by which the viscous sintering process of unit problems is simulated, is relatively recent. Generally, these numerical simulations are carried out by successively solving the Stokes problem for the present level geometry, and employing a time step to predict the next level geometry. The first numerical simulation of a unit problem of viscous sintering was carried out by Ross et al. [16]. They considered the sintering of an infinite line of equal cylinders and performed their simulation by employing a Finite Element Method (FEM). Unfortunately, they kept the center to center distance between those cylinders fixed (no shrinkage) which makes their study of less interest to our case.

Jagota and Dawson [17]-[19] were the first who could simulate some realistic unit viscous sintering problems. They applied the FEM to two axisymmetric problems, i.e. the coalescence of two equal spheres and of an infinite line of equal spheres. In Jagota and Dawson [18], the calculated behavior of the two coalescing spheres is used to simulate the densification of a powder compact. In that paper, the particle packing is modelled as a framework of links between any pair of touching spheres and the growth of those links is described by considering the behavior of each pair of coalescing spheres separately. Recently, Jagota [20] extended the model to simulate the viscous sintering of two equal glass coated spherical particles (two-phase sintering).

Kuiken [2] considered two-dimensional domains with a rather moderately varying curvature. He used an integral formulation based on the stream function and vorticity function and solved the resulting equations by employing a Boundary Element Method (BEM). The most important advantage of the BEM in comparison to the FEM is that the dimensionality of the problem is 
reduced by one, i.e. the two-dimensional fluid domain is replaced by a boundary curve which makes the moving boundary problem easier to solve from a computational point of view. Unfortunately, for the integral formulation that Kuiken applied, serious numerical problems occurred when simulating a geometry that shows a more extreme curvature. Those problems are due to inaccuracies in computing the derivative of the curvature, required in the chosen integral formulation (driving force).

In earlier work, cf. Van de Vorst et al. [21]-[26], we reported about the successful solution of the sintering problem for arbitrarily shaped two-dimensional finite fluid bodies with even pores inside. In those papers, the Stokes problem is described by another integral formulation based on boundary distributions of single- and double layer hydrodynamical potentials. The advantage of this formulation is that both the surface velocity and tension are the primary variables, i.e. after solving these equations the flow field for the surface is obtained directly. Moreover, a sophisticated (implicit) time integration scheme can be incorporated in the numerical scheme which allows to simulate the deformation of sharp curved geometries adequately. Using this solution method, the densification of all kinds of finite fluid domains can be simulated. However, due to the small size of such a pore domain compared to the whole gel structure, the mathematical model has to be advanced in order to get rid of effects in the densification simulations that can be contributed to the tension of the outer boundary of such a fluid domain. Because of this we require another, more advanced, mathematical model.

A more sophisticated approach to describe the sintering phenomenon is the determination of a representative unit cell within the gel and to consider the densification of it. This unit cell has to be chosen so that it reflects the sintering of the gel as a whole realistically. Such a unit cell may consist of a number of particles, depending on the microstructure; this cell is also referred to as a meso-cell (De With [27]). Examples of this approach are in fact the densification models developed by Mackenzie and Shuttleworth [7] and Scherer [8].

The model of Mackenzie and Shuttleworth [7] (MS-model) is generally accepted for the latestage viscous sintering. In this model, the densification results from the shrinkage of uniform spherical pores distributed throughout the gel. Hence the MS-model is also referred to as the closed pores model. The representative unit cell is an individual spherical pore for which the flow field can be calculated analytically. The MS-model leads to an equation for the sintering time necessary to reach a particular density of the gel.

Scherer [8] developed the so-called open pores model, which assumes the gel to be a regular three dimensional array of interconnected liquid cylinders, and considered its shrinkage. This model was used by Scherer to analyze the early and intermediate stage of the sintering of gels. For the unit cell that represents this structure, Scherer took a cubic array consisting of intersecting cylinders on all the edges from which the total surface was calculated (see also figure 2). After applying Frenkel's energy balance, he obtained an analytical relationship between the relative density and the time. However, the model breaks down when the pore is trapped in each cell, in the late sintering stage. Scherer's main result is a figure showing the density of the gel as a function of time which is very close to the predicted densification rate of the MS-model. In [9], Scherer extended his model to bimodal pore-sizes distributions. In [10], Scherer and Garino present an analysis for the sintering kinetics of porous glass layer on a rigid substrate (i.e. thin film coatings). 
In Van de Vorst [26], we presented a mathematical formulation which can be used to simulate the densification of a two-dimensional arbitrarily shaped unit cell numerically. Therefore, it is assumed that the structure of the gel can be described by a periodic continuation of this particular unit cell as time evolves; hence the unit cell has to be representative for the gel. The flow of the pores in the unit cell is described in terms of an integral formulation with kernels that incorporate the two-dimensional lattice assumption of the gel. It appears that the strength of the formulation is that one does not have to know the tension distribution on the boundary of the unit cell since this tension is cancelled out in the equations. In section 2 we briefly describe this mathematical model.

Using the above mentioned mathematical approach, we are able to examine the consequences of microstructure on the evolution of the pore size distribution. Here we will discuss the numerical findings obtained by using this formulation. The densification rate of one cylindrical pore in a square unit cell is compared with the analytic densification models of Mackenzie and Shuttleworth [7] and Scherer [8]. Next the densification phenomenon due to pore irregularities is considered for a nonuniformly sized cylindrical pores distribution which shows that the pores are vanishing in order of size one after another; the smallest pores first, followed by the larger ones. Moreover it will be shown that some of the pores may grow initially before they start shrinking at a later stage. Finally the densification of cylindrical packings is considered and the effect upon the densification rate when the cylindrical packing pore shape is replaces by a smooth pore shape.

\section{Mathematical Formulation and Numerical Solution for the Sintering of a Unit Cell}

Here, we briefly present the mathematical model in terms of an integral equation, to describe the sintering of a representative unit cell of a two-dimensional periodic lattice of Stokes fluid. More precise details about the derivation of this formulation can be found in Van de Vorst [26]. However, before we present this mathematical model, we will summarize the governing equations that describe the viscous sintering phenomenon.

The material transport by viscous sintering is modelled as a viscous incompressible Newtonian fluid driven solely by surface tension, cf. Kuiken [2]. So the Stokes creeping flow equations are valid, which can be characterized by the dynamic viscosity $\eta$, the surface tension $\gamma$ and the magnitude of the body say through its cross-section, e.g. length $\ell_{0}$. We define a characteristic velocity $v_{c}$, a characteristic pressure $p_{c}$ and a characteristic time $t_{c}$ based on the parameters $\gamma, \eta$ and $\ell_{0}$ by

$$
v_{c}=\frac{\gamma}{\eta}, \quad p_{c}=\frac{\gamma}{\ell_{0}}, \quad t_{c}=\frac{\ell_{0} \eta}{\gamma} .
$$

Using these characteristic parameters and taking $\ell_{0}$ as the characteristic length, we obtain for the Stokes equation the following dimensionless form

$$
\Delta \mathbf{v}-\operatorname{grad} p=0 .
$$




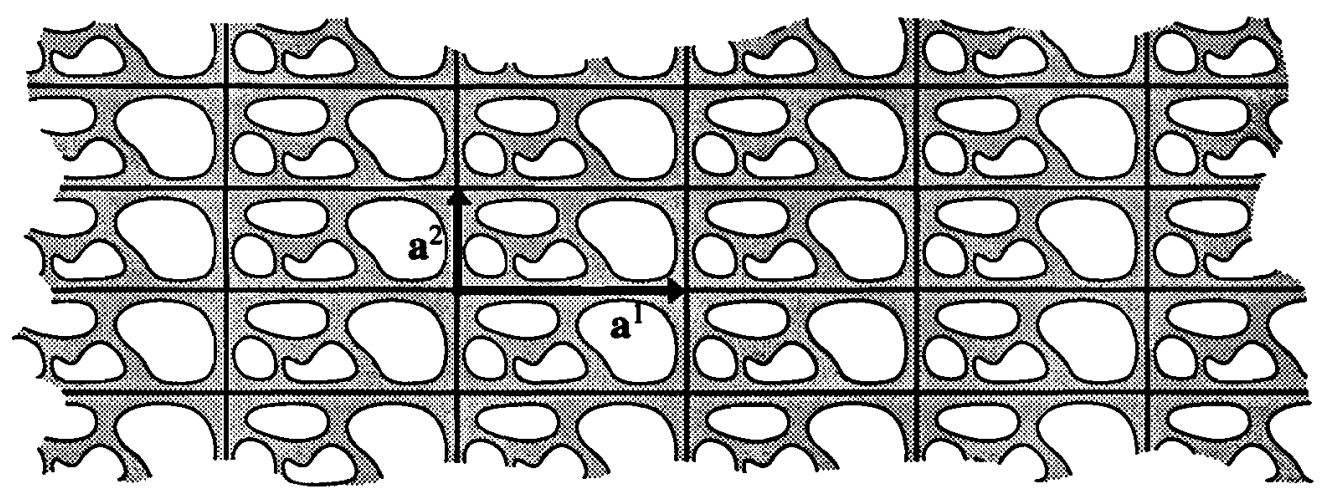

Figure 1: The representative unit cell and its basic vectors in a periodic two-dimensional Stokes fluid lattice.

Here $\mathbf{v}$ denotes the dimensionless velocity and $p$ is the dimensionless pressure of the flow. The conservation of mass can be expressed by the continuity equation

$$
\operatorname{div} \mathbf{v}=0
$$

The dimensionless stress tensor $\mathcal{T}$ for a Newtonian fluid is defined by

$$
\mathcal{T}_{i j}=-p \delta_{i j}+\left(\frac{\partial v_{i}}{\partial x_{j}}+\frac{\partial v_{j}}{\partial x_{i}}\right) .
$$

On the boundary, the dimensionless tension in the normal direction, say $\mathbf{b}$, for a free fluid surface can be found as (Batchelor [28, p.150])

$$
b_{i}:=\mathcal{T}_{i j} n_{j}=-\kappa n_{i}
$$

where $\kappa$ is the curvature of the boundary and $\mathbf{n}$ is the outward unit normal vector.

The motion of the boundary is obtained by applying the Lagrangian representation for the boundary velocity $\mathbf{v}$,

$$
\frac{\mathrm{d} \mathbf{x}}{\mathrm{d} t}=\mathbf{v}(\mathbf{x}), \quad(\mathbf{x} \in \Gamma),
$$

where $t$ is the dimensionless time. The kinematic constraint above expresses the displacement of the material boundary particles: the trajectories of those particles are followed. Hence a quasi-static approach is used to solve the viscous sintering problem.

The important assumption that we use in the mathematical model presented here, is that we suppose that the sintering gel can be represented by a periodic continuation, in two directions, of a unit cell with a certain pores distribution at any stage during the densification process (cf. figure 1). So the shrinkage of that particular unit cell corresponds to the shrinkage rate of the whole gel in the two-dimensional plane. In fact, this is the same assumption which is used by the derivation of the analytical models of both Mackenzie and Shuttleworth [7] and Scherer [8] as are mentioned in the introduction.

Let $\mathbf{a}^{1}$ and $\mathbf{a}^{2}$ be the time dependent basic vectors of the unit cell of the lattice (see figure 1). Hence the position of the $\lambda^{\text {th }}$ lattice is given by

$$
\mathbf{X}^{\lambda}=\lambda_{1} \mathbf{a}^{3}+\lambda_{2} \mathbf{a}^{2} \quad\left(\lambda_{i}=0, \pm 1, \pm 2, \ldots\right) \text {. }
$$


Denote by $\Gamma_{0}$ the outer boundary of the unit cell and let $\Gamma_{1} \ldots \Gamma_{M}$ be all the pore boundaries. Moreover, let $\mathbf{x}$ be a point on the boundary of a pore. Then, the following integral formulation for the velocity of the pores in the unit cell can be derived,

$$
\frac{1}{2} v_{j}(\mathbf{x})+\sum_{m=0}^{\mathbf{M}} \int_{\Gamma_{m}} q_{i j}(\mathbf{x}-\mathbf{y}) v_{j}(\mathbf{y}) d \Gamma_{y}=\sum_{m=1}^{\mathbf{M}} \int_{\Gamma_{m}} u_{i j}(\mathbf{x}-\mathbf{y}) b_{j}(\mathbf{y}) d \Gamma_{y} .
$$

Here a repeated index in an expression denotes a summation over all possible values of that index (Einstein summation convention) and $d \Gamma_{y}$ denotes that the integral is considered with respect to the vector $\mathbf{y}$. The kernels $q_{i j}(\mathbf{x})$ and $u_{i j}(\mathbf{x})$ are given by the following expressions,

$$
\begin{aligned}
& q_{i j}(\mathbf{x})= \frac{x_{i} n_{j}}{\tau}+\frac{1}{2|\tau|} \sum_{\lambda}\left[-r_{j}^{\lambda} n_{i}-\delta_{i j} r_{k}^{\lambda} n_{k}+\frac{2 r_{i}^{\lambda} r_{j}^{\lambda} r_{k}^{\lambda} n_{k}}{\boldsymbol{r}^{2}}\left(1+\frac{|\tau|}{\pi \boldsymbol{r}^{2}}\right)\right] \exp \left(-\frac{\pi \boldsymbol{r}^{2}}{|\tau|}\right) \\
&+\frac{1}{\tau} \sum_{\mu}^{\prime} \frac{1}{k^{4}}\left[\left(1+\frac{|\tau| k^{2}}{4 \pi}\right)\left(k^{2}\left(K_{j}^{\mu} n_{i}-\delta_{i j} K_{k}^{\mu} n_{k}\right)-2 K_{i}^{\mu} K_{j}^{\mu} K_{k}^{\mu} n_{k}\right)\right. \\
&\left.+k^{2} K_{i}^{\mu} n_{j}\right] \exp \left(-\frac{|\tau| k^{2}}{4 \pi}\right) \sin \left(\mathbf{x} \cdot \mathbf{K}^{\mu}\right) .
\end{aligned}
$$

and

$$
\begin{aligned}
u_{i j}(\mathbf{x})= & \frac{\delta_{i j}}{8 \pi}\left[\log \left(\frac{\tau}{\pi}\right)-2-\gamma\right] \\
& -\frac{1}{8 \pi} \sum_{\lambda}\left[\delta_{i j} \mathrm{E}_{1}\left(\frac{\pi r^{2}}{\tau}\right)+2\left(\frac{r_{i}^{\lambda} r_{j}^{\lambda}}{\boldsymbol{r}^{2}}-\delta_{i j}\right) \exp \left(-\frac{\pi \boldsymbol{r}^{2}}{\tau}\right)\right] \\
& +\frac{1}{4 \pi \tau} \sum_{\mu}^{\prime} \frac{1}{k^{4}}\left(-\delta_{i j} k^{2}+K_{i}^{\mu} K_{j}^{\mu}\right)\left(4 \pi+\tau k^{2}\right) \exp \left(-\frac{\tau k^{2}}{4 \pi}\right) \cos \left(\mathbf{x} \cdot \mathbf{K}^{\mu}\right),
\end{aligned}
$$

where $\cdot$ is the inner product, $\mathrm{E}_{1}(x)$ denotes the Exponential integral, which is defined by

$$
\mathrm{E}_{1}(x)=\int_{1}^{\infty} e^{-x t} t d t
$$

and $\gamma=0.5772156649 \ldots$ is Euler's constant. The other variables $r_{j}, r$ and $k$ in equations (2.8) and (2.9) are subsequently equal to $r_{j}^{\lambda}=x_{j}-X_{j}^{\lambda}, \boldsymbol{r}=\left|\mathbf{x}-\mathbf{X}^{\lambda}\right|$ and $k=\left|\mathbf{K}^{\mu}\right|$, where $\mathbf{K}^{\mu}$ is a vector in the so-called reciprocal lattice space and is denoted by

$$
\mathbf{K}^{\mu}=\mu_{1} \mathbf{b}^{1}+\mu_{2} \mathbf{b}^{2} \quad\left(\mu_{i}=0, \pm 1, \pm 2, \ldots\right) .
$$

The vectors $\mathbf{b}^{1}$ and $\mathbf{b}^{2}$ are the basic vectors of the unit cell of the reciprocal lattice which are defined as

$$
\mathbf{b}^{1}=\frac{2 \pi}{\tau}\left(a_{2}^{2},-a_{1}^{2}\right)^{\mathbf{T}}, \quad \mathbf{b}^{2}=\frac{2 \pi}{\tau}\left(-a_{2}^{1}, a_{1}^{1}\right)^{\mathbf{T}},
$$

and $\tau$ is the total surface area of one cell, i.e. $\tau=a_{1}^{1} a_{2}^{2}-a_{1}^{2} a_{2}^{1}$. Note that the following relation is satisfied between the basic vectors of the original and the reciprocal lattice,

$$
\mathbf{a}^{i} \cdot \mathbf{b}^{j}=2 \pi \delta_{i j} .
$$


Moreover, we remark that when the kernel of equation (2.9) is considered as a velocity field $u_{i}^{k}=u_{i k}$, then this is the fundamental solution of the flow due to a lattice of point forces in the $\mathbf{e}^{k}$ direction in the two-dimensional plane.

The integral equation (2.7) is solved by applying a Boundary Element Method (BEM). Therefore, the boundary is discretized into a set of nodal points and the boundary curve is replaced by a polygon through these nodal points. The integral formulation is enforced on this polygon for each of the collocation points. This results into a square full rank system of linear algebraic equations for the unknown velocity in the discretization points. The motion of the boundary is obtained by applying the kinematic constraint (2.5) for each collocation point into the system of equations, which results into a system of non-linear ODEs. It appears that this system of ODEs can be stiff for certain type of shapes (e.g. shapes which are having cusp-like regions). Therefore, we have implemented a variable step, variable order Backward Differences Formulae (BDF) method to solve these ODEs. More details about the implementation of the BEM and this time integration scheme are available in Van de Vorst [24] and Van de Vorst and Mattheij [25].

\section{Numerical Results}

Results of numerical simulation for a number of two-dimensional sintering geometries are presented to compare the numerical densification rate with the analytic shrinkage models and to show some typical evolution properties. All the calculations are performed on a SiliconGraphics Indy and/or Power Challenge with R4000 and R8000 processor respectively. The CPU time required to compute the various problems presented below, varied between 10 minutes and a couple of days.

\subsection{Analytic Shrinkage Models Compared to Numerical Simulations}

One important issue in sintering research is the quantification of the rest-porosity of the gel after sintering. The scientific interest for this densification process is to understand the magnitude of the driving force for this process and to deduce how the driving force and thus the densification rate are affected by the gel microstructure. As we mentioned in the introduction, the gel microstructure is depending on the gelation process and preparation conditions. In this subsection we briefly overview three analytical shrinkage relationships which have been applied in literature to predict the densification rate of a shrinking gel. We then compare these models to the numerical simulation results of two-dimensional shapes with pores inside. Although a number of these models are developed for a really three-dimensional sintering compact, the comparison with the numerical results give some quantitative insight in the reliability and limitations of those approximations.

The first approach we discuss is the model of Mackenzie and Shuttleworth [7] for the latestage viscous sintering of a compact (MS-model). As we mentioned in the introduction, this formulation is also referred to as the closed pores model. The densification results from the shrinkage of spherical pores distributed throughout the compact. Moreover, it is assumed that all 


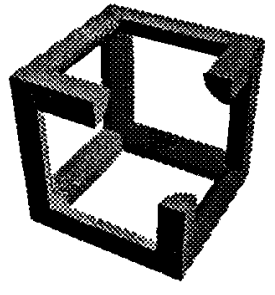

a.

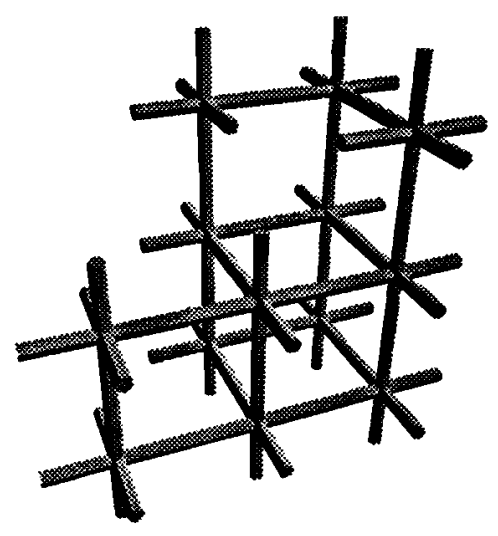

b.

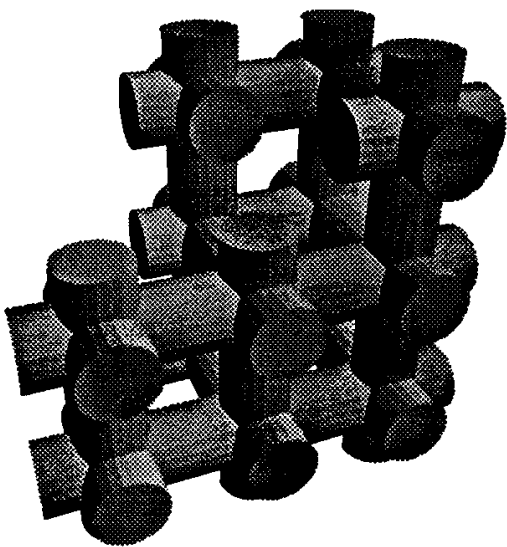

c.

Figure 2: Microstructural model of a gel developed by Scherer [8] consisting of liquid cylinders in a cubic array: (a) is the unit cell of the microstructure, (b) and (c) are a low and a high density gel structure respectively.

the pores have an identical radius. The velocity field of such a pore can easily be obtained and after applying Frenkel's energy balance (cf. section 1) one derives an analytical relationship between the relative density of the compact and the reduced time. The MS-model in full dimensional variables gives the following densification rate

$$
K_{3}\left(\mathrm{t}-\mathrm{t}_{0}\right)=\frac{2}{3}\left(\frac{3}{4 \pi}\right)^{\frac{1}{3}} \int_{\hat{\rho}_{0}}^{\hat{\rho}} \frac{d s}{(1-s)^{\frac{2}{3}} s^{\frac{1}{3}}}
$$

where

$$
K_{3}=\frac{\gamma n^{\frac{1}{3}}}{\eta} ; \quad \hat{\rho}_{0}=\frac{\rho_{0}}{\rho_{s}} ; \quad \hat{\rho}=\frac{\rho}{\rho_{s}} \quad \text { with } \quad 0<\hat{\rho}_{0} \leq \hat{\rho}<1 .
$$

Here $\rho$ is the bulk density of the gel, $\rho_{0}$ is the initial bulk density (at $t=t_{0}$ ), $\rho_{s}$ is the density of the solid phase (skeletal density) and $n$ is the number of closed pores per unit of volume of solid phase. After integration of the right-hand side of the equation above, we obtain

$$
K_{3}\left(\mathrm{t}-\mathrm{t}_{0}\right)=\frac{2}{3}\left(\frac{3}{4 \pi}\right)^{\frac{1}{3}}\left(\frac{1}{2} \log \left[\frac{\left(1+a_{0}\right)^{3}\left(1+a^{3}\right)}{(1+a)^{3}\left(1+a_{0}^{3}\right)}\right]+\sqrt{3} \arctan \left[\frac{2 \sqrt{3}\left(a_{0}-a\right)}{3+\left(2 a_{0}-1\right)(2 a-1)}\right]\right),
$$

where

$$
a_{0}=\left(\frac{1-\hat{\rho}_{0}}{\hat{\rho}_{0}}\right)^{\frac{1}{3}} \quad \text { and } \quad a=\left(\frac{1-\hat{\rho}}{\hat{\rho}}\right)^{\frac{1}{3}} .
$$

In the two-dimensional plane, we can deduce an analogous MS-model by assuming that the densification results from the shrinkage of uniform cylindrical pores distributed throughout the fluid. The derivation of this shrinkage equation is similar to the outline of Mackenzie and 
Shuttleworth [7] where the pores are modelled by spheres. The following densification rate is then obtained

$$
\begin{aligned}
K_{2}\left(\mathrm{t}-\mathrm{t}_{0}\right) & =\frac{1}{\sqrt{\pi}} \int_{\hat{\rho}_{0}}^{\hat{\rho}} \frac{d s}{\sqrt{s(1-s)}} \\
& =\frac{1}{\sqrt{\pi}}\left[\arcsin \left(1-\hat{\rho}_{0}\right)-\arcsin (1-\hat{\rho})\right],
\end{aligned}
$$

where $K_{2}=\gamma \sqrt{n} / \eta$. In the sequel, we will refer to relation (3.1) as the 3D MS-model and to relation (3.2) as the 2D MS-model.

The second approach we describe is the so-called open-pores model of Scherer [8] which is used to analyze the early and intermediate stage of the sintering process. As was stated in the introduction, in this description it is assumed that the gel can be modelled as a regular three-dimensional array of interconnected liquid cylinders, see also figure 2. For the unit cell representing this structure, a cubic is taken which is characterized by the edge length and the cylinder radius (figure 2a). After approximating the flow field of this unit cell and using Frenkel's energy balance the following equation can be deduced

$$
K_{3}\left(\mathrm{t}-\mathrm{t}_{0}\right)=\frac{1}{2^{\frac{1}{6}}}\left(\frac{1}{2} \log \left[\frac{\left(1-b_{0}+b_{0}^{2}\right)(1+b)^{2}}{\left(1-b+b^{2}\right)\left(1+b_{0}\right)^{2}}\right]+\sqrt{3} \arctan \left[\frac{2 \sqrt{3}\left(b_{0}-b\right)}{3+\left(2 b_{0}-1\right)(2 b-1)}\right]\right),
$$

where

$$
b=\left(\frac{2\left(1+\cos \frac{1}{3} \varphi\right)}{1-2 \cos \frac{1}{3} \varphi}\right)^{\frac{1}{3}} ; \quad \varphi=\arctan \left[\frac{8 \sqrt{2 \hat{\rho}\left(\pi^{3}-32 \hat{\rho}\right)}}{\pi^{3}-64 \hat{\rho}}\right]+\left\{\begin{array}{cc}
\pi & \hat{\rho}<\pi^{3} / 64 \\
2 \pi & \hat{\rho}>\pi^{3} / 64
\end{array}\right.
$$

and $b_{0}$ is found by substituting $\varphi_{0}$ in the relation for $b$. The variable $\varphi_{0}$ is similarly defined by using $\hat{\rho}_{0}$ instead of $\hat{\rho}$ in the equation above. Equation (3.3) applies for $0 \leq \hat{\rho} \leq 0.942$ only, since it can be shown that at the density $\hat{\rho}=0.942$ the parallel cylinders of the unit cell touch. Next, each cell contains an isolated pore so that the MS-model applies. Note that the equation presented above is not equal to the analytical form as was deduced in Scherer [8]. After some analytical manipulation, his rather complicated equation(s) can be rewritten as (3.3). The equation in its present form shows much similarity with the relation obtained with the MS-model.

The third approach we mention is an extension of Scherer's open-pores model to describe the densification rate of a gel on a rigid substrate. This model is developed in Scherer and Garino [10] and represents the gel by cylindrical tubes whose axes are normal to the substrate. Hence this model may probably more apply to two-dimensional sintering problems like the ones we consider here. In [10] the following analytical relationship between the density and the reduced time is given,

$$
K_{3}\left(\mathrm{t}-\mathrm{t}_{0}\right)=\frac{2}{\hat{\rho}_{0}^{\frac{1}{3}} \sqrt{\pi}}\left(3\left(\sqrt{1-\hat{\rho}_{0}}-\sqrt{1-\hat{\rho}}\right)+2 \arctan \left[\frac{\sqrt{1-\hat{\rho}_{0}}-\sqrt{1-\hat{\rho}}}{1+\sqrt{\left(1-\hat{\rho}_{0}\right)(1-\hat{\rho})}}\right]\right),
$$

where $\hat{\rho}$ may vary between 0 and 1 .

The dimensionless form of all analytical densification equations presented above can easily be found by skipping the factor $\gamma / \eta$ and taking $\rho_{s}=1$, hence $\hat{\rho}=\rho$. In order to show the applicability 


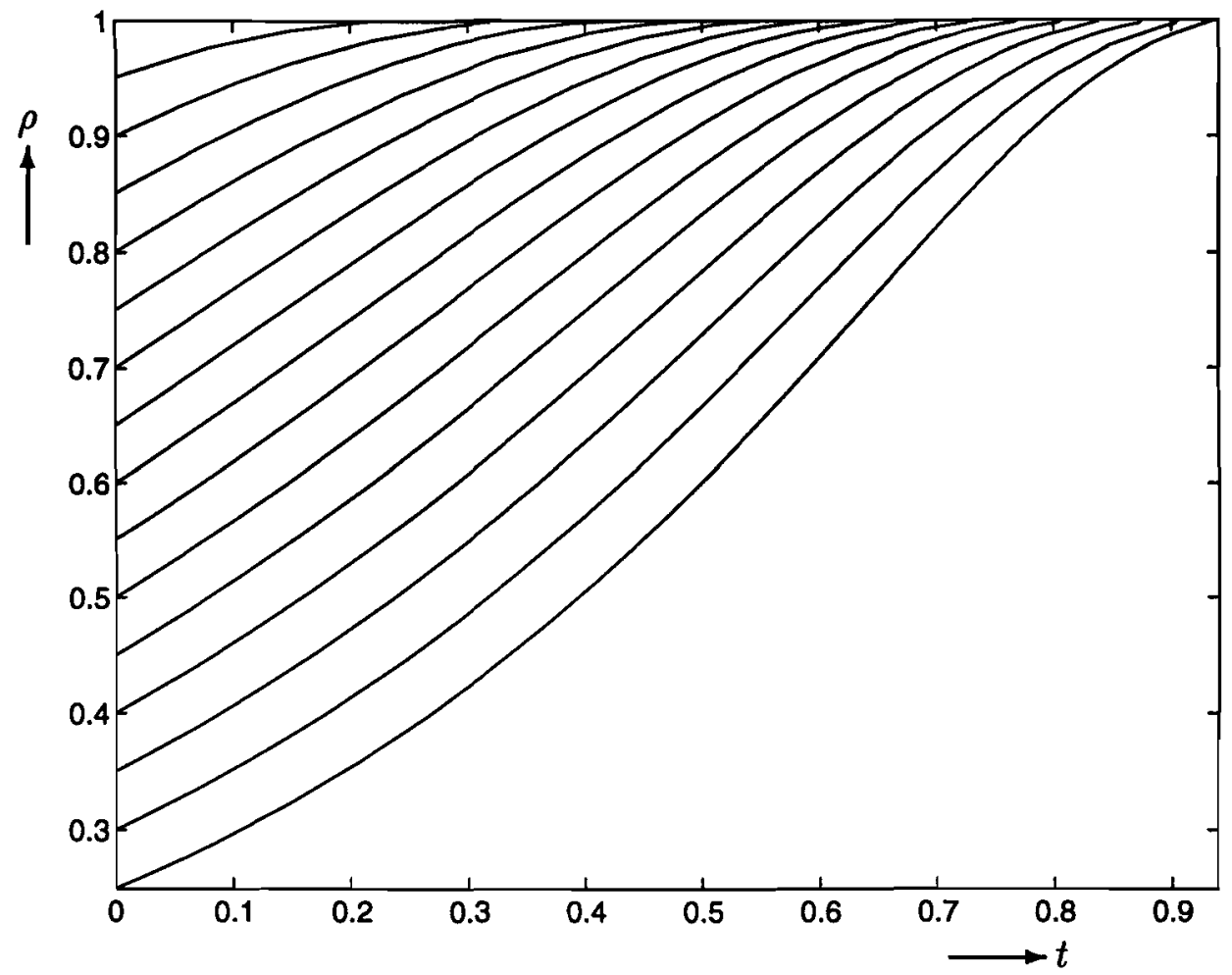

Figure 3: The densification rate obtained for one cylindrical pore in a 1 by 1 unit cell by varying the initial radius of the pore.

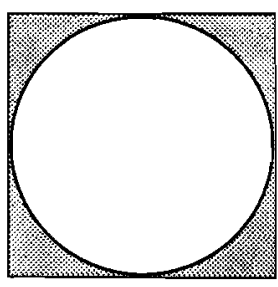

$t=0.0$

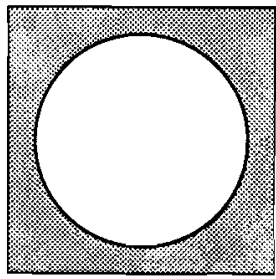

$t=0.0$

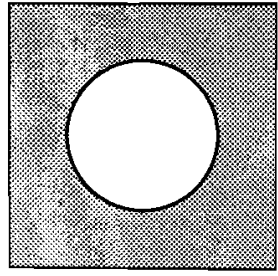

$t=0.0$

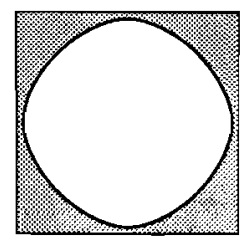

$t=0.2$

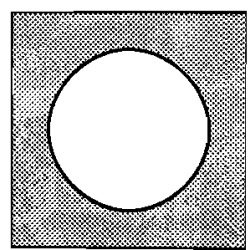

$t=0.2$

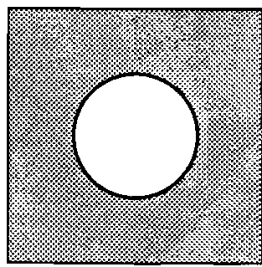

$t=0.1$

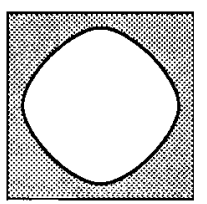

$t=0.4$

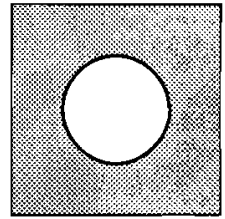

$t=0.4$

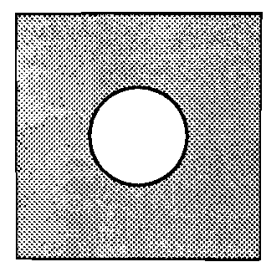

$t=0.2$

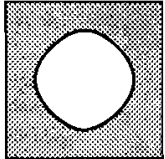

$t=0.6$

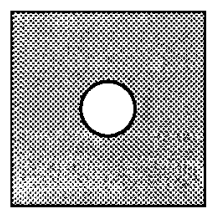

$t=0.6$

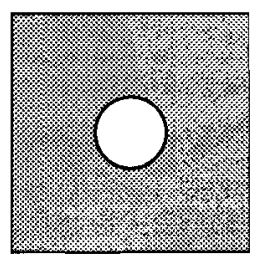

$t=0.3$ $t=0.8$

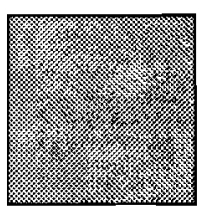

$t=0.8$
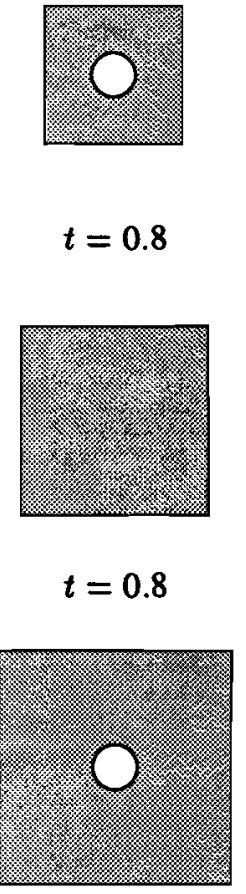

$t=0.4$

Figure 4: The shape deformation of some of the above used unit cells at subsequent time steps. The initial density $\rho_{0}$ of these cells is equal to $0.25,0.5$ and 0.75 respectively. 

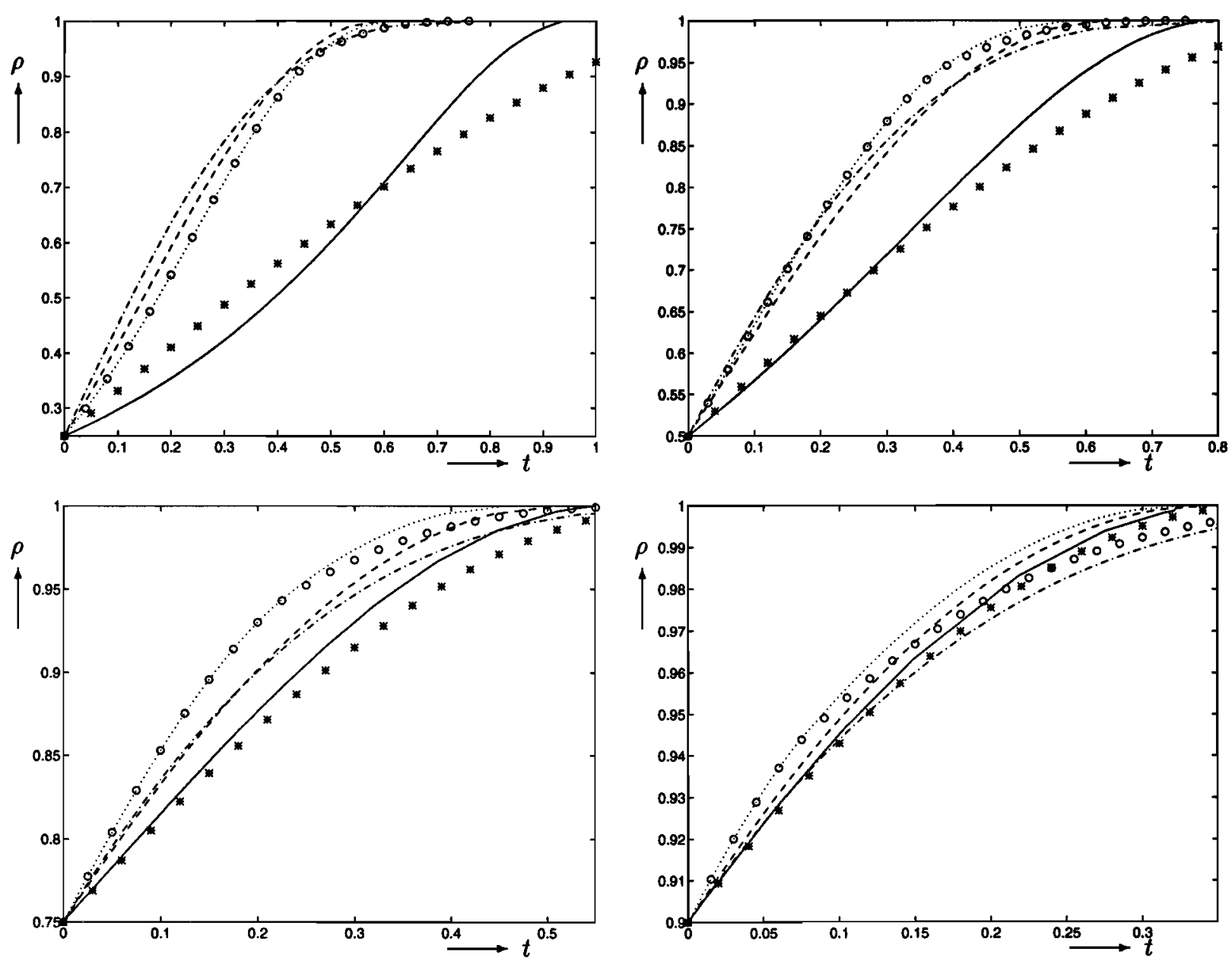

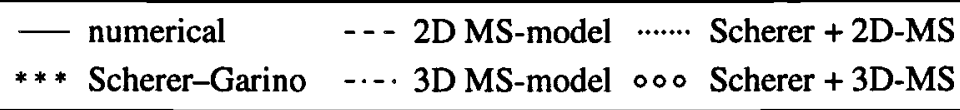

Figure 5: The comparison of the analytic predicted densification rates from several models with the numerically obtained density change of various unit cells with one cylindrical pore and initial density $\rho_{0}$ equal to $0.25,0.5,0.75$ and 0.9 respectively.

of the above described (actually) three-dimensional models to two-dimensional problems, we consider a fluid with cylindrical pores of equal radius which are distributed uniformly in the two-dimensional plane. Thus we consider the same geometrical conditions as are assumed in the development of some of the above described analytic densification models.

The initial size of the square unit cell is taken equal to 1 and the radius of the inner pore is varied to obtain a particular initial density. The density $\rho$ of the numerically calculated fluid domain at a particular time is found from dividing the surface occupied by fluid in the unit cell by the total area that surrounds the outer boundary of the cell. In figure 3 the numerically obtained densification rates are plotted of the unit cells with initial density $\rho_{0}=0.25(0.05) 0.95$.

The differences in densification rates of the various unit cells at later time stages of the 


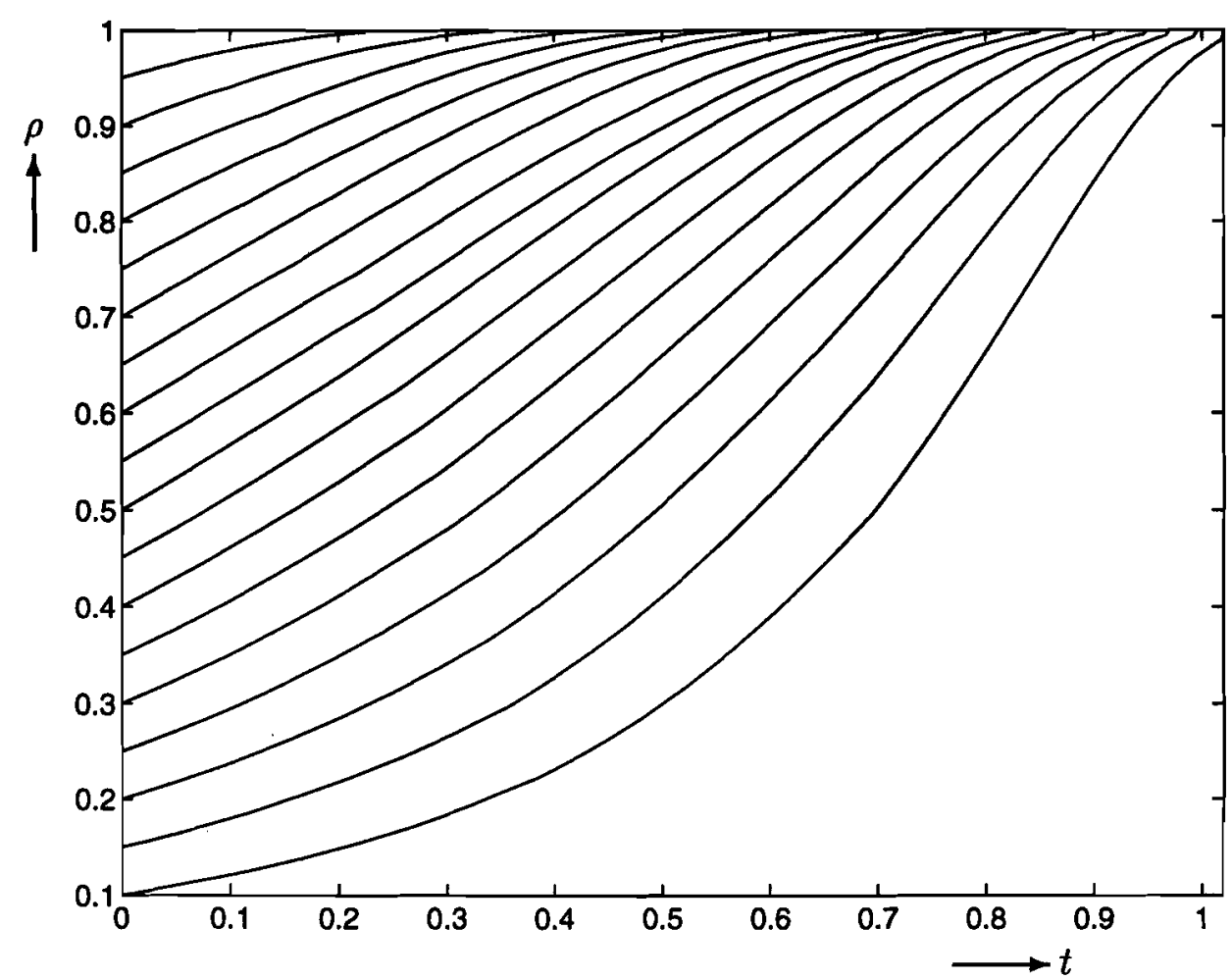

Figure 6: The densification rate obtained for one square pore with rounded-off corners in a 1 by 1 unit cell by varying the initial size of the pore.

sintering process, as can be observed in figure 3, are due to the fact that the amount of fluid for each unit cell differs. This results from taking the initial size of all the outer cell boundaries the same. Hence, we conclude that the densification rate depends on both the size of the pore and its distribution in the fluid, i.e. the size of the unit cell. This is quite logically from a physical point of view.

The shape deformation of some of the used unit cells is plotted in figure 4 at subsequent time steps. Here the initial density of the showed cells is taken equal to $0.25,0.5$ and 0.75 respectively. It can be seen that for the shapes with a relatively high initial density, the densification process proceeds by a circular shrinkage of the cylindrical pores. On the other hand at lower initial densities, the influence of the pores onto each other results into a more rounded-off square shaped deformation during the early stage. However, the latter effect does not seem to influence the densification rate during the early stage which can also be observed in figure 3 .

In figure 5 the numerically obtained density change for four of the above considered unit cells is compared with the density increase as predicted by the analytical relations discussed previously, i.e. the relations (3.1)-(3.4). The initial density $\rho_{0}$ of the unit cell of these four plots is subsequently equal to $0.25,0.5,0.75$ and 0.9 . In order to apply the analytic relations, we have to take $\rho_{0}$ equal to the starting density at $t_{0}=0$. The number $n$ is equal to the total number of pores (1) divided by the surface occupied by fluid. Moreover, Scherer's equation (3.3) has to be coupled with the MS-model to make this relation applicable for all densities. Therefore, we have used both the 2D and 3D MS-models, i.e. equations (3.2) and (3.1) respectively. 


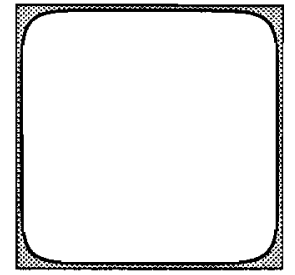

$t=0.0$

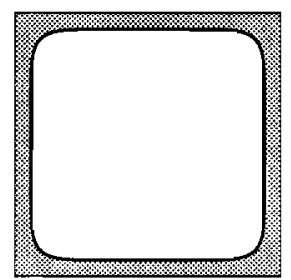

$t=0.0$

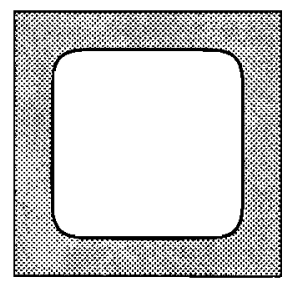

$t=0.0$

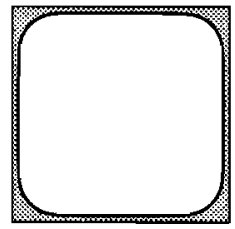

$t=0.2$

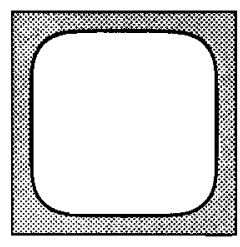

$t=0.2$

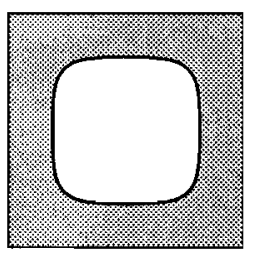

$t=0.2$

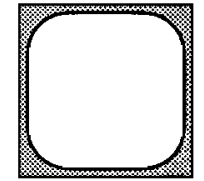

$t=0.4$

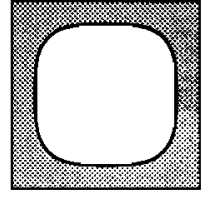

$t=0.4$

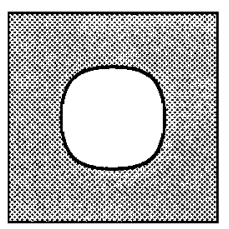

$t=0.4$

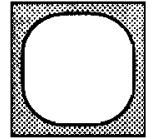

$t=0.6$

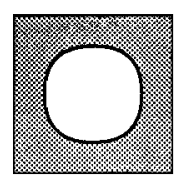

$t=0.6$

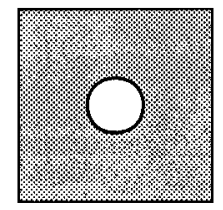

$t=0.6$

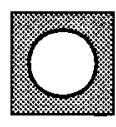

$t=0.8$

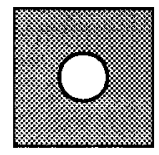

$t=0.8$

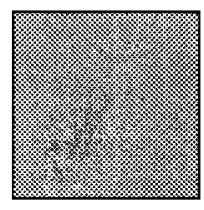

$t=0.8$

Figure 7: The shape deformation of some of the in figure 6 used unit cells at subsequent time steps. The initial density $\rho$ of these cells is equal to $0.1,0.25$ and 0.5 .

From the various plots shown in figure 5 it can be observed that the analytic predictions become far better for increasing initial density. In particular, the densification rate predicted by the Scherer-Garino equation (3.4) shows a very good likeness with the numerical results at initial densities higher than 0.75 . This is not surprising since this model is based on a uniformly sized and distributed cylindrical pores geometry and the starting geometry is likewise. The 2D MS-model (3.2) is also based on such a pores distribution, but the matching with the numerical results is only sufficiently at very high initial densities. However, this is still a satisfactory result since in practice it is known that the MS-model only holds during the late stage of the sintering process. From the observation that both the models developed for a two-dimensional geometry predict the densification of a cylindrical pores distribution with a relatively high initial density $(\rho>0.75$ ) quite well, i.e. the Scherer-Garino equation and the 2D MS-model, we may conclude that the three-dimensional models from which these two equations are extensions, i.e. Scherer's equation and the 3D MS-model respectively, should also give satisfactory results in the three-dimensional case.

However an important observation from figure 5 is that all considered analytic relations fail considerably in the prediction of the densification rate of the cells with a low or intermediate initial density. Note that still the best results are obtained by using the Scherer-Garino equation. An explanation for this failure might be the fact that the shrinkage of each individual pore is influenced by the shrinking of the neighboring pores. This effect is not included in the densification models 


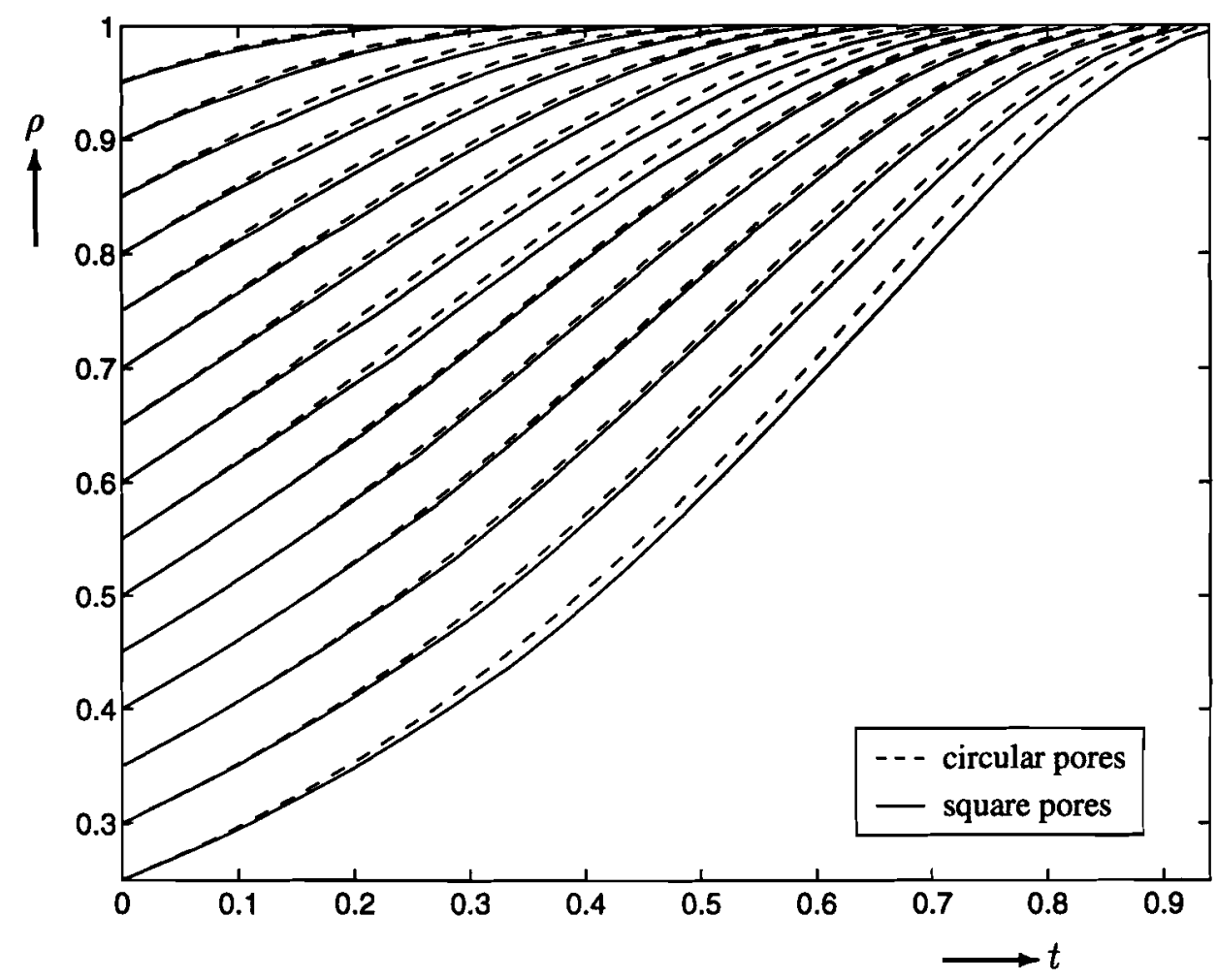

Figure 8: A good matching is shown when comparing both the numerically obtained densification rates for the circular and rounded-off square pores distributions. This suggests that the knowledge of the exact pore shape is of less importance in actual situations.

discussed above.

For two-dimensional viscous fluids with initial density lower than 0.25 , it is not possible to use a cylindrical pores distribution since the pores do not fit within the unit cell. Because of this we consider a square pore with rounded-off corners in a square unit cell of length 1 . The initial pore shape is obtained by using the function $x^{8}+y^{8}=a$, where $a$ is a constant which is taken such that a prescribed initial cell density is obtained. In figure 6 we have plotted the numerically found densification rate for cells with initial density $\rho_{0}=0.1(0.05) 0.95$.

Some typical shape deformations of these unit cells are shown in figure 7 for the geometries where the initial density $\rho_{0}$ is taken equal to $0.1,0.25$ and 0.5 respectively. From the shape deformation of the cell with initial density $\rho_{0}=0.1$, it can be observed that again the deformation is effected by the neighboring pores like in the cylindrical pores case. Moreover, as the figure suggests, the higher the initial density of the cell the faster the square pore deforms towards a cylindrical pore. This latter effect can also be demonstrated when comparing the densification rates obtained for the cylindrical pores distribution (figure 3 ) with the results found for the square pores distribution (figure 6) as are plotted in figure 8. It can be seen that a good matching is obtained between the shrinkage rates of both pore shapes. Hence this suggests that the knowledge of the exact pore shape is of less importance, which is observed in actual situations too. 


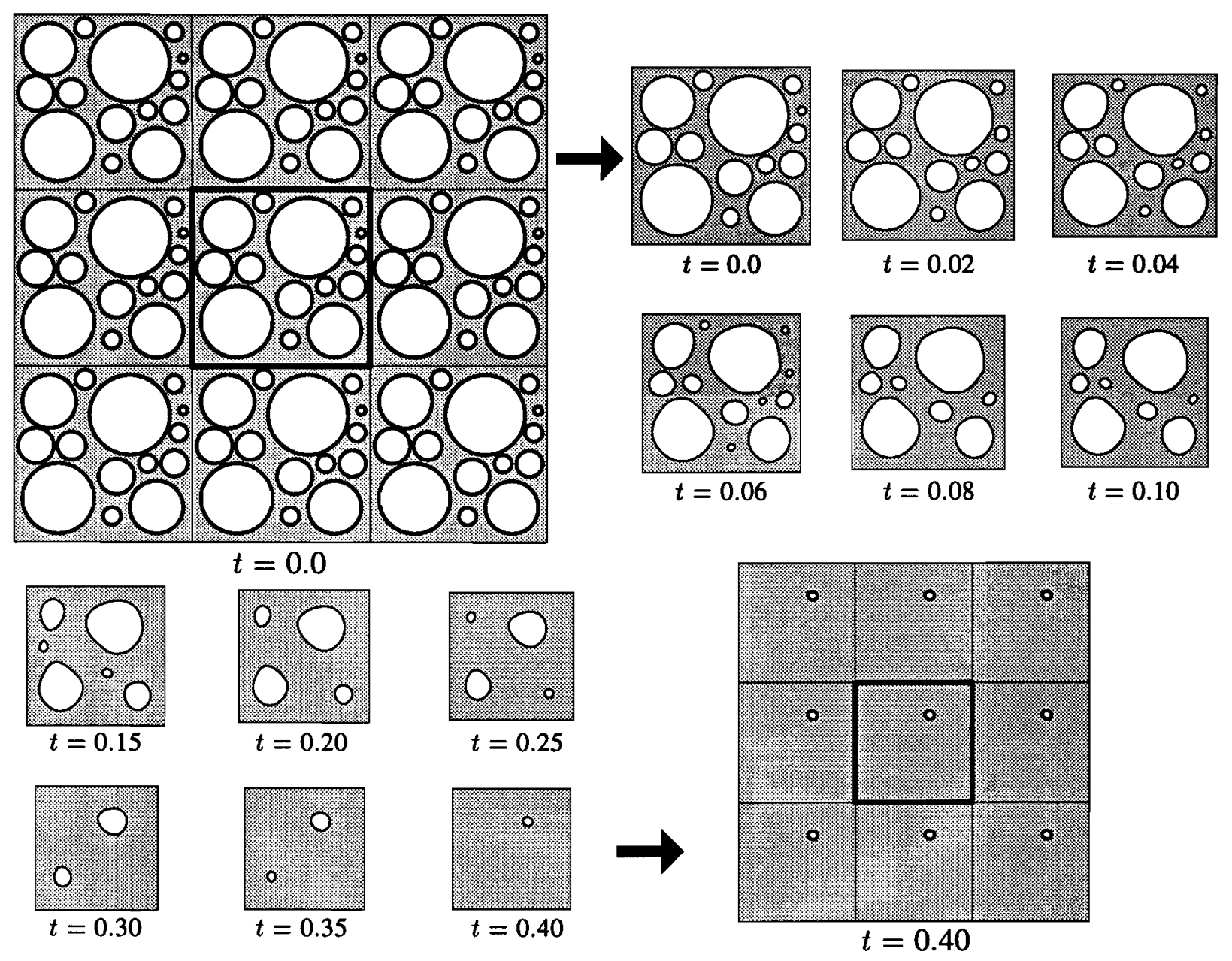

Figure 9: The shape deformation of the unit cell of a periodic lattice of non-uniformly sized cylindrical pores at subsequent times demonstrates that the pores vanish in order of size.

\subsection{Densification Effects due to Pore Irregularities}

In practice, one does not deal with a uniform sized pore distribution as we considered in the previous subsection. Usually, the sintering compact is an irregular particle packing that consists of a variety of particle sizes with (often) a non-spherical-(cylindrical) form. Moreover another effect due to these irregularities has to be taken into account: the rearrangement of particles and the opening and closure of pores induced by this rearranging of particles.

The effect of nonuniformly sized and distributed pores can be illustrated by the fluid lattice plotted in figure 9 at $t=0.0$. Here we consider a unit cell of length 1 by 1 with 14 nonuniformly sized cylindrical pores from which the radii are varying between 0.025 and 0.225 . The plots at various time steps of the deformed cell shape show that the pores vanish in order of size one after another: all pores are shrinking which results in the vanishing of the smallest pores first, followed by the larger pores. Moreover, we observe from these pictures that the aspect ratio of the unit cell is changing which might be seen as an effect on the shrinkage of the entire viscous 


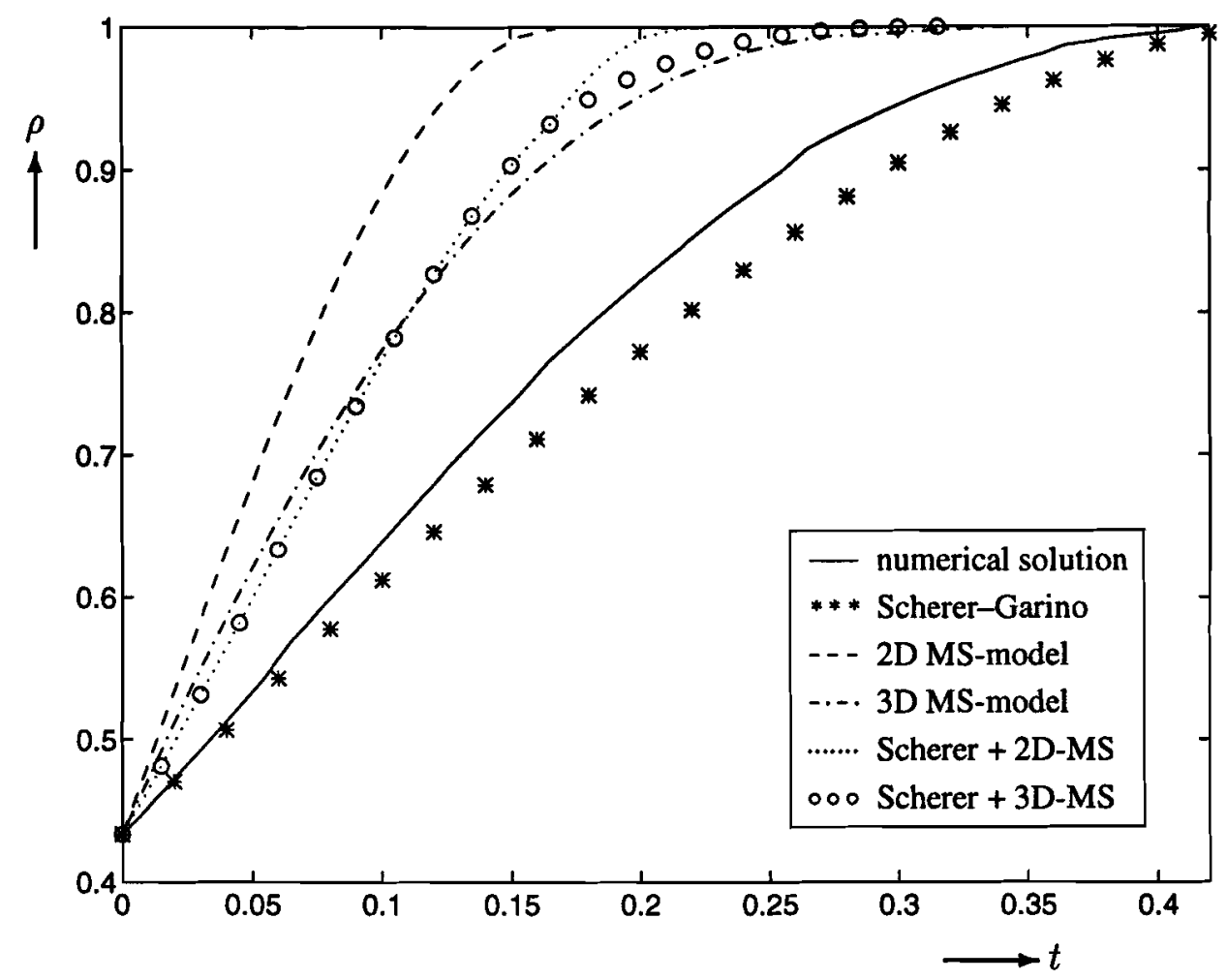

Figure 10: The numerical densification rate of the periodic lattice of non-uniformly sized cylindrical pores of figure 9 is compared with the various densification model predictions. A surprisingly good matching is obtained between the numerical solution and the Scherer-Garino prediction. However, one should not take a general conclusion from this observation.

sintering body.

This behavior is the opposite of the numerical results obtained for a finite two-dimensional fluid region with nonuniform sized pores (cf. Van de Vorst [24, p.117]). There, it appears that the initially larger sized pores are shrinking significantly faster as compared to the smallest ones as time evolves. This difference can be explained from the fact that in the finite domain case, the fluid obtains an extra tension due to the curvature of the outer boundary.

In figure 10 the numerically obtained densification rate of the above unit cell is plotted which is compared to the various densification models that are discussed in the previous subsection, i.e. equations (3.1)-(3.4). These relations are applied by taking into account all the pores of the unit cell, thus both the smaller and larger pores (12). Hence the variable $n$ in the densification relations, i.e. the number or pores per unit of volume of fluid, is equal to $n=14 / 0.433=32.3$. Again we observe that the Scherer-Garino equation provides the most accurate prediction of the numerically obtained rate. For this example the matching of the latter two densification rates is surprisingly good; however, this observation should not be generalized as will be illustrated by the following example.

An important issue of the sintering process is to deduce how the driving force and thus the densification rate are affected by the microstructure, i.e. what kind of structural configuration 
(a)

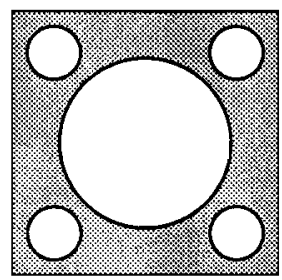

$t=0.0$

(b)

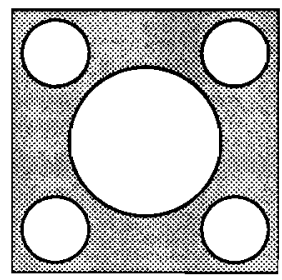

$t=0.0$

(c)

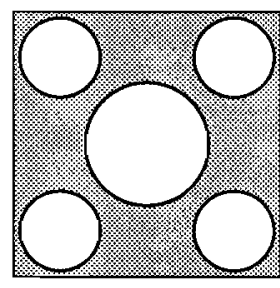

$t=0.0$

(d)

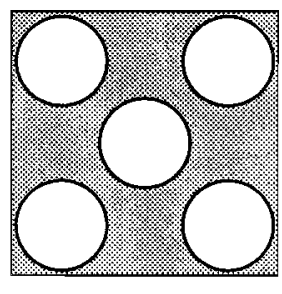

$t=0.0$

(e)

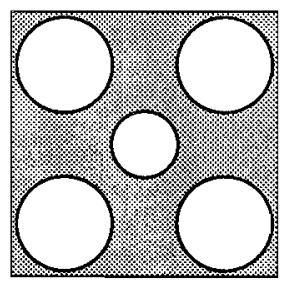

$t=0.0$

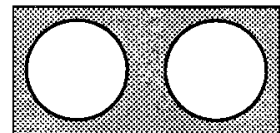

(f)

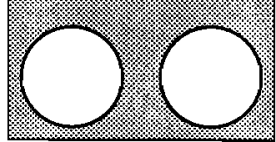

$t=0.0$

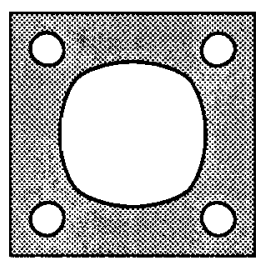

$t=0.15$

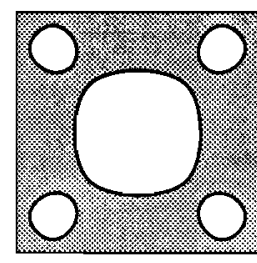

$t=0.15$

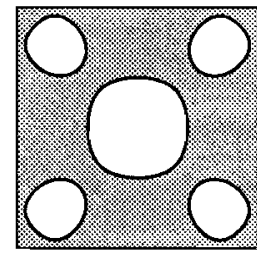

$t=0.15$

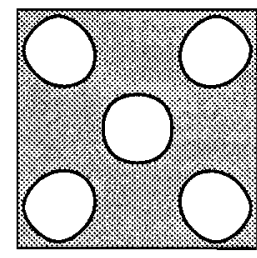

$t=0.15$

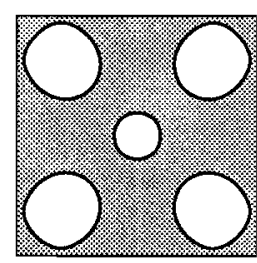

$t=0.15$

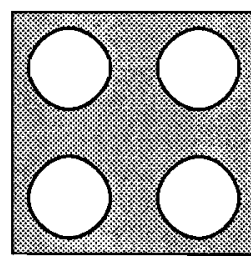

$t=0.15$

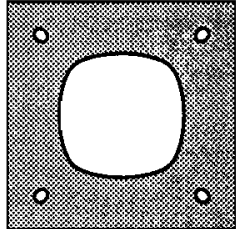

$t=0.3$

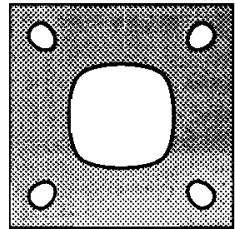

$t=0.3$

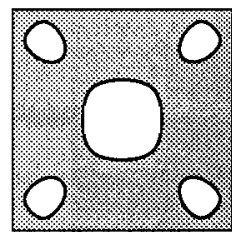

$t=0.3$

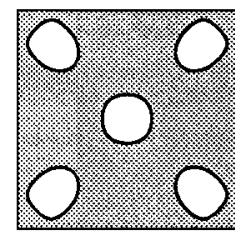

$t=0.3$

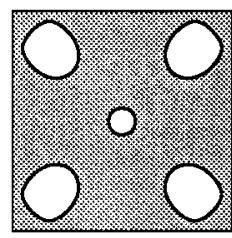

$t=0.3$

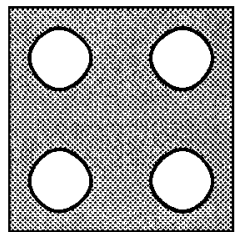

$t=0.3$

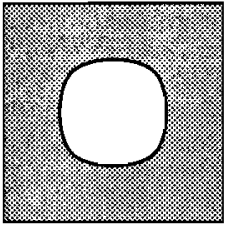

$t=0.45$

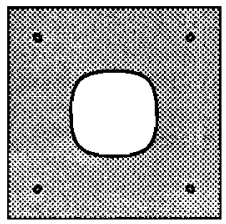

$t=0.45$

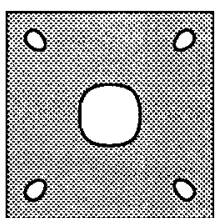

$t=0.45$

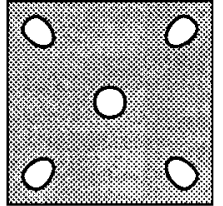

$t=0.45$

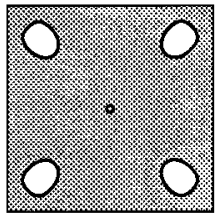

$t=0.45$

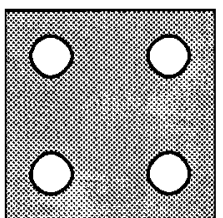

$t=0.45$

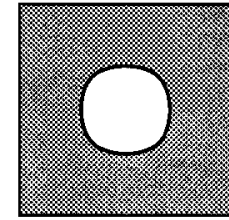

$t=0.6$

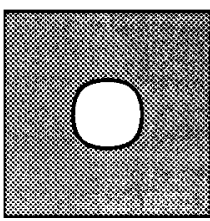

$t=0.6$

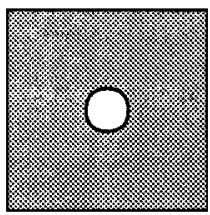

$t=0.6$

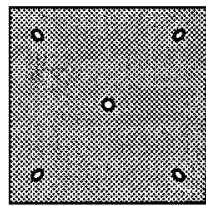

$t=0.6$

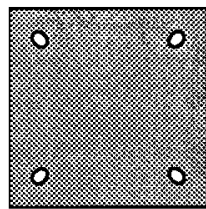

$t=0.6$

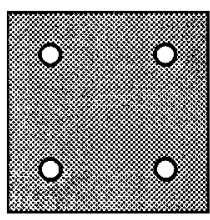

$t=0.6$

Figure 11: The shape deformation at subsequent time steps of six 2 by 2 unit cells with a non-uniformly sized circular pores distribution and an equally initial density of 0.55 , demonstrates the various densification behavior. 


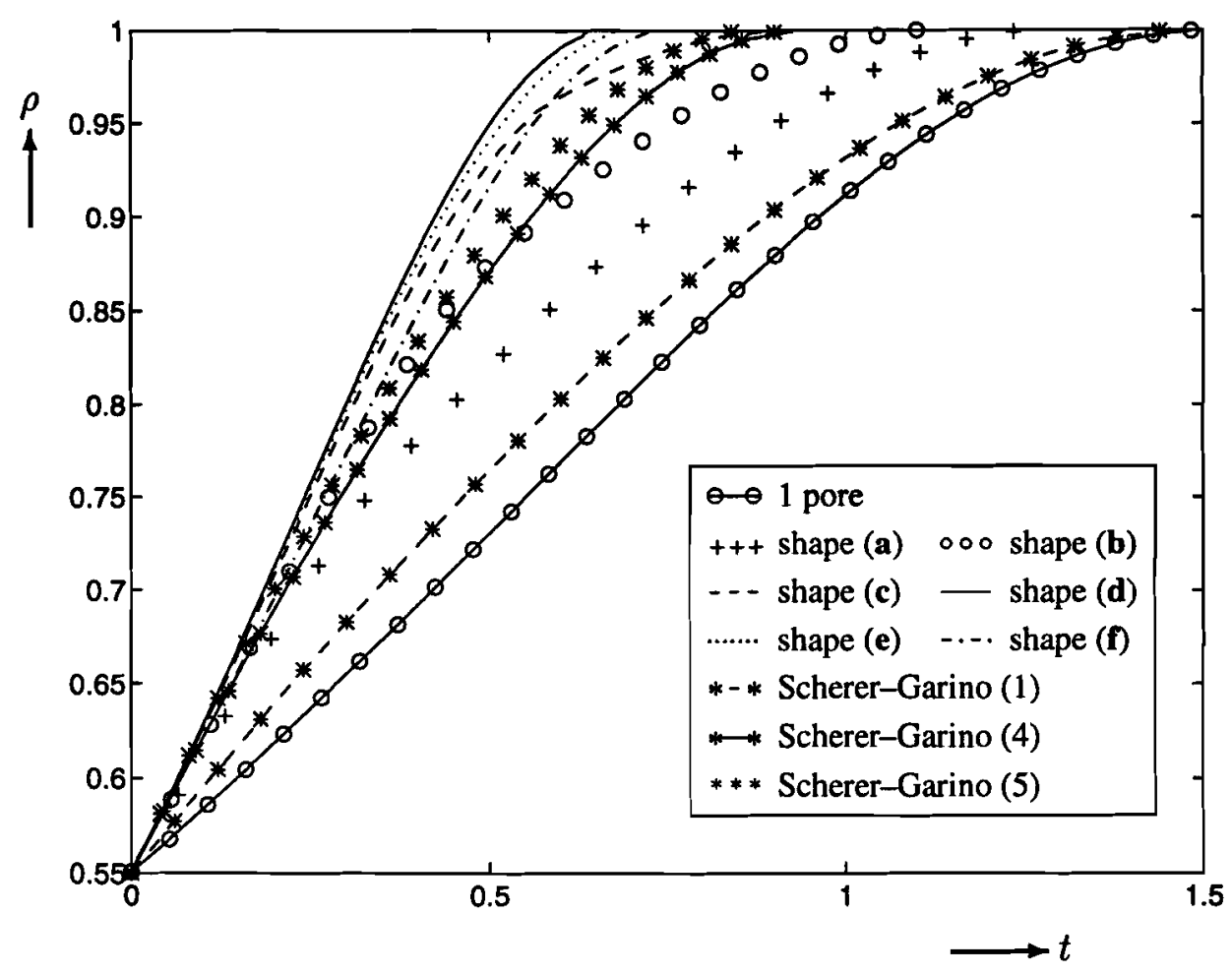

Figure 12: The density $(\rho)$ change of both the periodic lattices of arbitrary cylinders of figure 11 for increasing time $(t)$ is compared with the Scherer-Garino equation for the case that in this prediction 1, 4 or 5 pores are taken inside the unit cell. The variety of rates demonstrates the limitations of the relation.

leads to a higher free energy of the gel and hence to a higher densification rate. Here, we demonstrate the dependence of the pore size distribution on the densification rate of an initially 2 by 2 unit cell with one, four or five cylindrical pores. The center point of one cylindrical pore is placed in the center of the cell; four other equally sized cylindrical pores are symmetrically situated along the diagonals of the unit cell. The radii of all the pores are varied with respect to the restriction that the initial density $\rho_{0}$ of each cell is equal to 0.55 .

In figure 11 the shape deformations for six different configurations (a)-(f) are plotted at five subsequent time stages, i.e. $t=0.0(0.15) 0.6$. As can be observed from this picture, the density reached at $t=0.6$ differs quite a lot between the various start configurations. The densification rate of all these cells are compared in figure 12. In this figure the density change is included for the case that the cell consists of only one single pore too. The time lasted to obtain a fully dense cell varies between 0.63 for shape (d), i.e. five equally sized pores, and 1.48 for the one pore geometry. Hence the densification of the latter geometry proceeds 2.4 times slower than shape (d).

In this figure, we have also plotted the densification rates as are obtained by applying the Scherer-Garino relation when 1, 4 or 5 pores are taken inside the unit cell. Now, these relations do not correspond well to the numerically obtained rates as was the case in the previous example shape (e.g. figure 10). Hence the example shapes of figure 11 are demonstrating the limitations of 


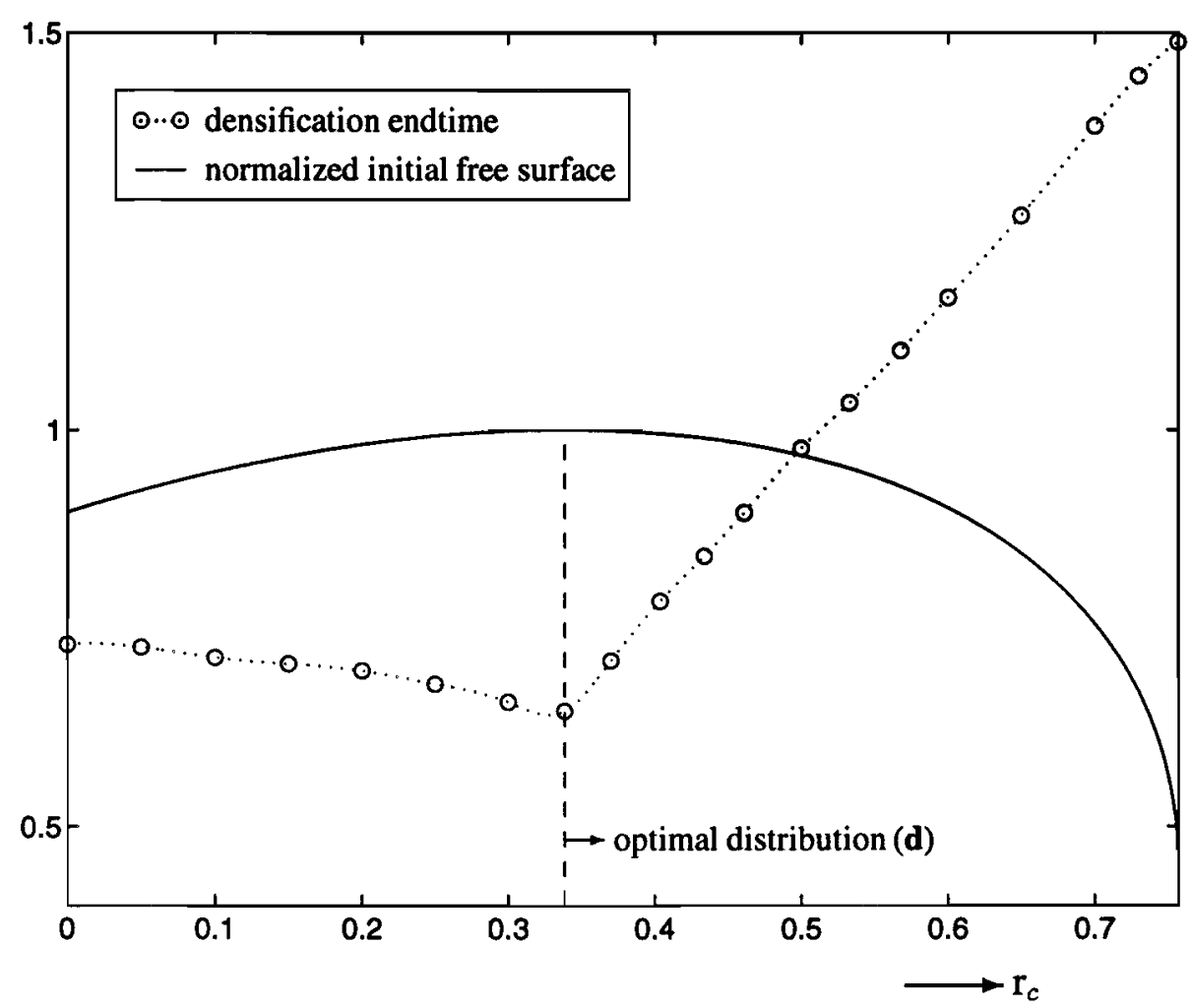

Figure 13: The importance of the pore size distribution is demonstrated by varying the radius of the center pore $\left(r_{c}\right)$ for the start configuration type of shape of figure 11. The densification end time behaves at both the sides of the optimal distribution (d) quite different. This is in contrast to the normalized initial free surface energy which is only varying slightly in the neighborhood of the optimum.

this prediction: something about the pore size distribution should be incorporated in the analytic densification relation in order to improve such a model.

From numerical experiments, it appears that distribution (d) is even the optimum start geometry, i.e. all other configurations are leading to a higher densification end time. This is also demonstrated by figure 13 . There $r_{c}$ denotes the the radius of the cylindrical pore that is situated in the center of the unit cell. This radius $r_{c}$ can be varied between the bounds 0 and 0.7569 . In the figure, the bullets denote the computed densification end time when the starting unit cell, with a certain central pore radius $r_{c}$, is completely filled by fluid. i.e. $\rho=1$. As we already mentioned, the optimal (minimal) densification end time is reached when all the pores are initially of equal size, i.e. distribution (d) where $r_{c}=0.33839$. The latter observation is not surprising since for this type of configuration it can be proven that geometry (d) corresponds to the geometry with the largest total length of the boundary pores, i.e. the largest initial free surface energy, which is illustrated in figure 13 too. There we have plotted by a solid line the normalized free surface, i.e. the total length of the starting pore boundary by varying the radius $r_{c}$, which is divided through the total length of the optimal start geometry (d). Note that the initial pore boundary length of distribution (d) is equal to $3.3839 \pi=10.631$.

From the figure it can be observed that the total initial boundary length does not change much 

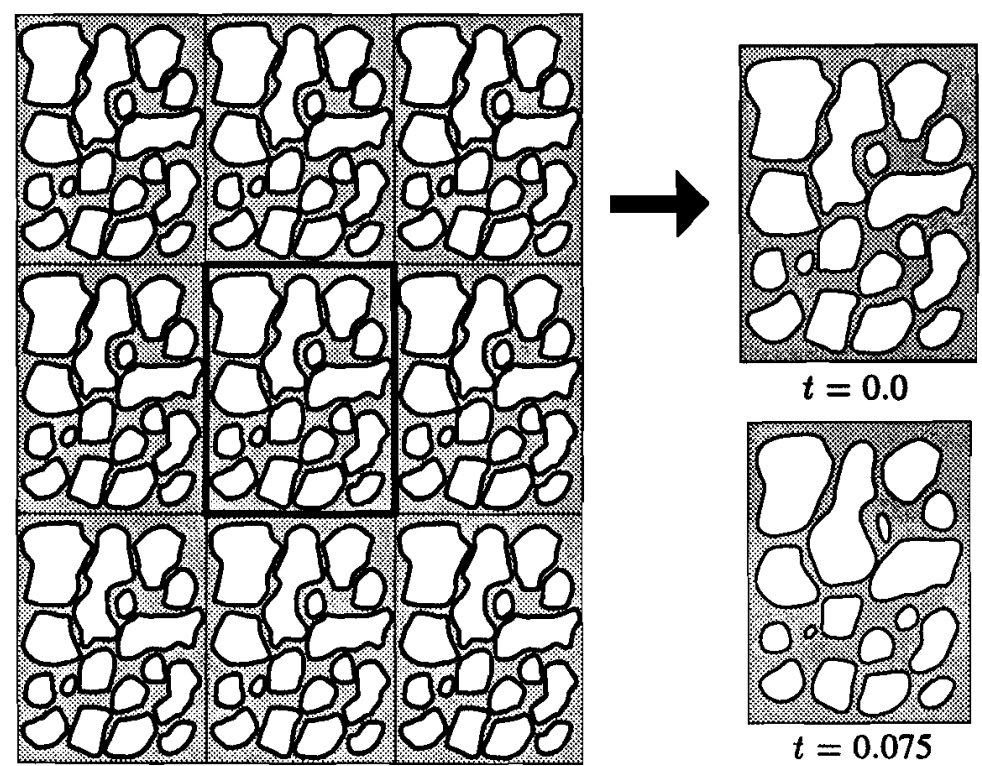

$t=0.0$
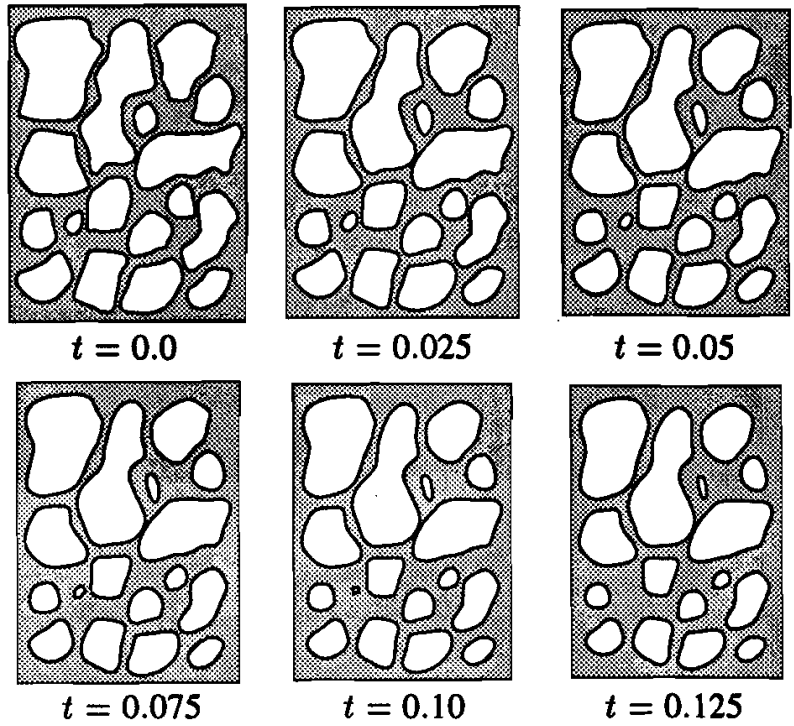

$t=0.05$
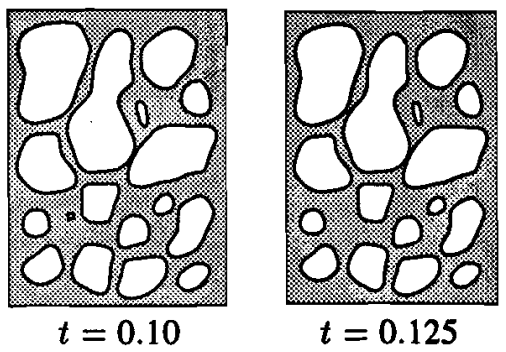

$t=0.0$

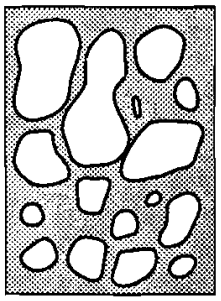

$t=0.15$

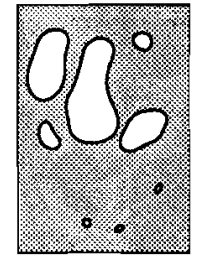

$t=0.45$

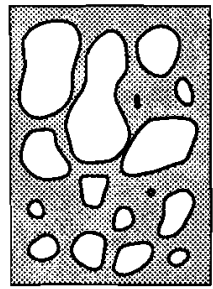

$t=0.20$

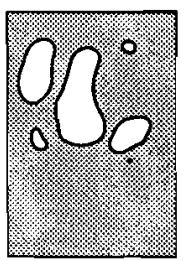

$t=0.50$

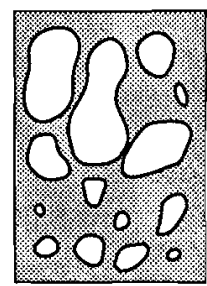

$t=0.25$

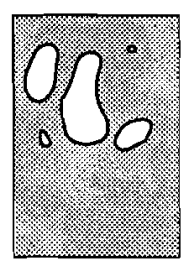

$t=0.55$

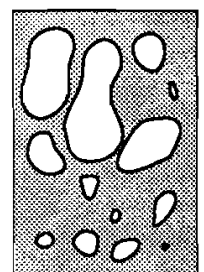

$t=0.30$

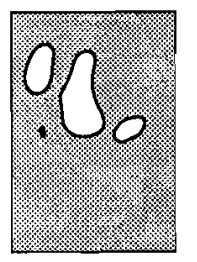

$t=0.60$

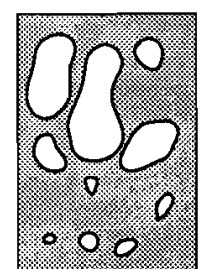

$t=0.35$

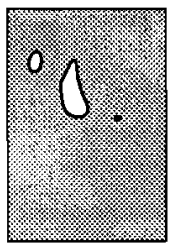

$t=0.80$

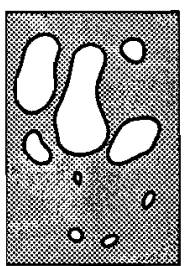

$t=0.40$

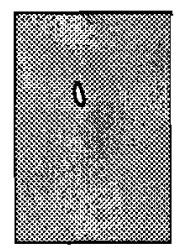

$t=1.0$

Figure 14: The densification of a texture model of a base-catalysed aerogel demonstrates that some pores are growing initially before they start shrinking. This phenomenon influences the densification rate negatively.

when the radius of the center pore varies between 0.0 and 0.6. However, the opposite holds for the densification end time which behaves quite differently at both the sides of the optimal configuration (d). When the center pore becomes smaller than the other four pores $\left(r_{c}<0.338\right)$, this leads to a small increase of the densification end time only. On the other hand when the radius of the center pore is increased, the densification end time increases dramatically, even for a small increase of $r_{c}$ near the optimum. The latter effect is quite surprising when taking into consideration that the total initial free surface at both sides of the optimum is nearly equivalent for a substantially long range of $r_{c}$. It seems that the pore size distribution is of more importance with respect to the densification rate than the initial free surface of the pores distribution. The different behavior in densification rate at both sides of the optimum distribution (d) might be 


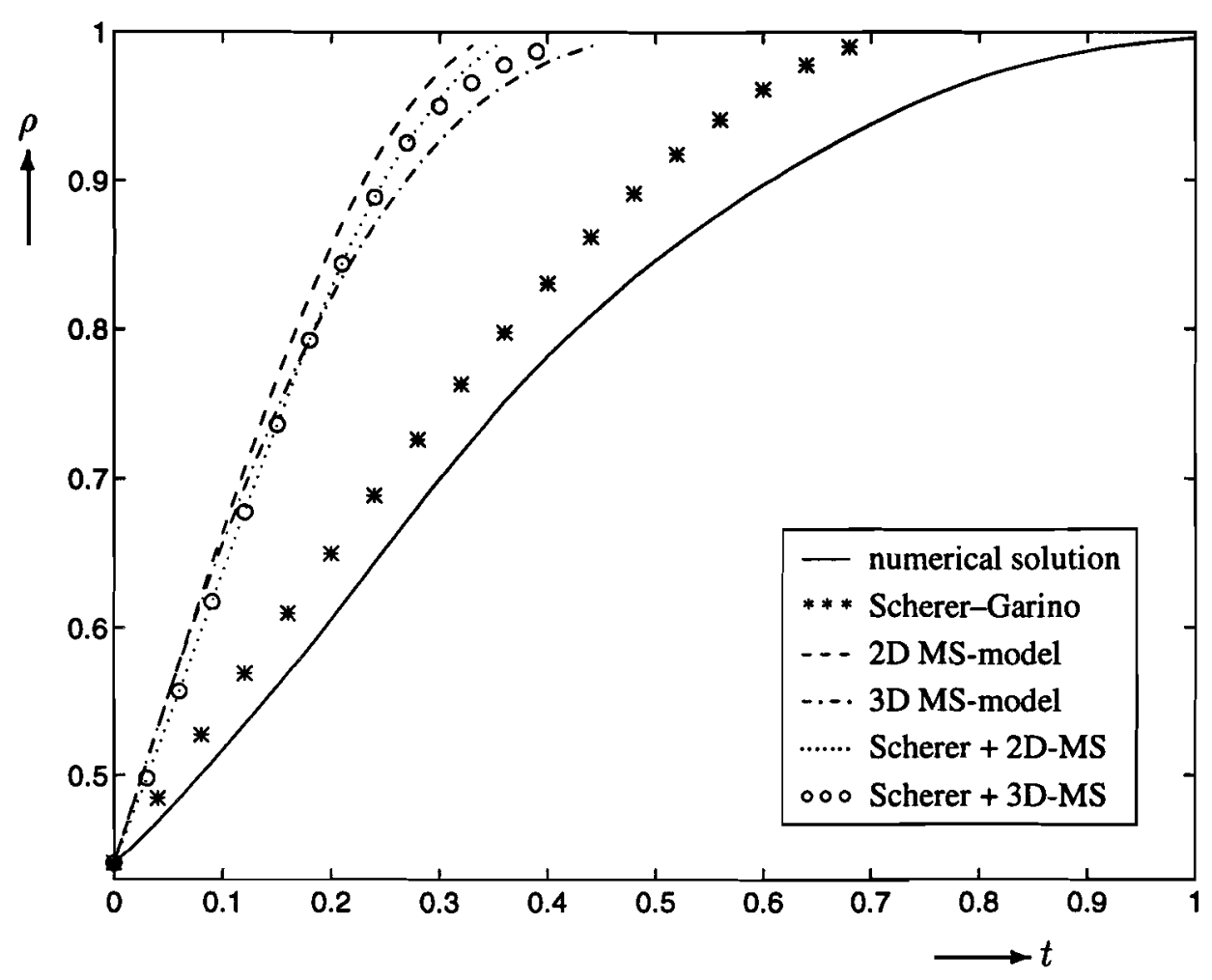

Figure 15: The numerically obtained densification rate of the aerogel texture model of figure 14 is compared with the various analytical predictions. Again, the Scherer-Garino equation provides the best approximation.

explained from the observation that the pores are vanishing in subsequent order of size, i.e. first the smallest pores followed by the larger ones. In the case that $r_{c}<0.338$, there is only one smaller pore so that after the vanishing of this pore, there will be four pores left with a total pore boundary length (e.g. free surface energy) that will be much larger than in the case that $r_{c}>0.338$; then four small pores are vanishing so that only one pore remains.

All pores in the cells we considered so far were shrinking during the entire densification process. However, it might also be possible that some pores first grow in size, before they shrink and vanish. This process will influence the densification rate negatively. In figure 14 this phenomenon is demonstrated. The unit cell represents a texture model of an aerogel that is formed in a base-catalysed way (after Craievich et al. [29]). A characteristic of the matrix material, i.e. the skeletal material, of an aerogel produced in such a way is that it contains closed micropores; the material itself contains meso- and macro-pores. Here we have ignored the micropores in the skeletal material. This approach is justified since during the first stage of the sintering process, the micropores are eliminated by polycondensation reactions (cf. Craievich et al. [29]). Because of these reactions, this stage is dominated by diffusion which results in an increase of the skeletal density. Thereafter, the meso- and macro-pores are eliminated by coalescence and densification through a viscous flow. Here we investigate the shrinkage of irregular sized pores distributions of such a microstructure model with an already relatively high initial density. Another model for the microstructure of a base-catalysed aerogel with a low initial density will be considered 


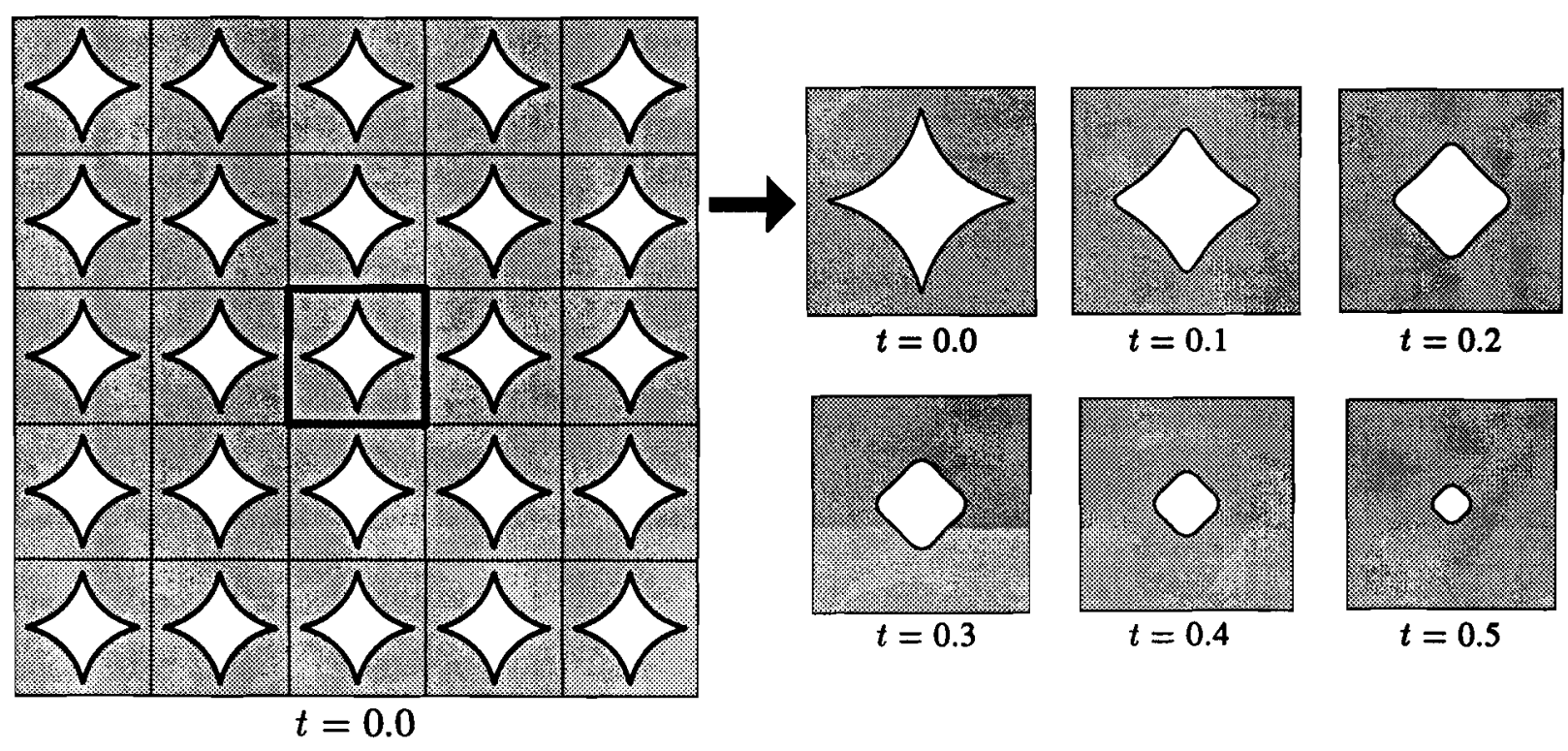

Figure 16: The shape deformation of the unit cell of a regular cylindrical particle packing at subsequent time stages.

in the next subsection. Initially, the size of the rectangular unit cell is taken equal to 2.1 by 2.8 , with density $\rho_{0}=0.439$ and it contains 17 pores. Another model for the microstructure of a base-catalysed aerogel with a low initial density will be considered in the next subsection.

Again it can be seen from the subsequent time plots in figure 14 that the smaller pores are vanishing first. Moreover, we observe that during the early time stage some pores become larger in size before they start shrinking. Especially this occurs for pores which have a large concave boundary part, which explains the possible grow of such a pore by noting that the pore has a much longer boundary length than it would strictly require to surround the pore contents. Hence, due to the "excess" of boundary, the pore may expand whereas the total pore boundary length still decreases as time evolves. Thus we conclude that one should avoid such kind of pore shapes as much as possible.

The density change of the unit cell of the aerogel texture model is plotted in figure 15 for increasing time by a solid line. In the same figure we have also plotted the results obtained from the analytical predictions. Again, we observe that the Scherer-Garino equation provides the most accurate prediction of the numerical densification rate. It seems to be impossible to introduce the phenomenon of growing pores in such a densification model due to the geometrical dependence; so the only way to discover this effect for a certain given microstructure would be by numerical simulation of the representative unit cell.

\subsection{Densification of cylindrical packings}

The microstructure of colloidal silica gels and xerogels are normally modelled by packing configurations of spherical particles or clusters. Because of this we demonstrate here some densification properties of the two-dimensional analoque of such a microstructure, i.e. geometries 


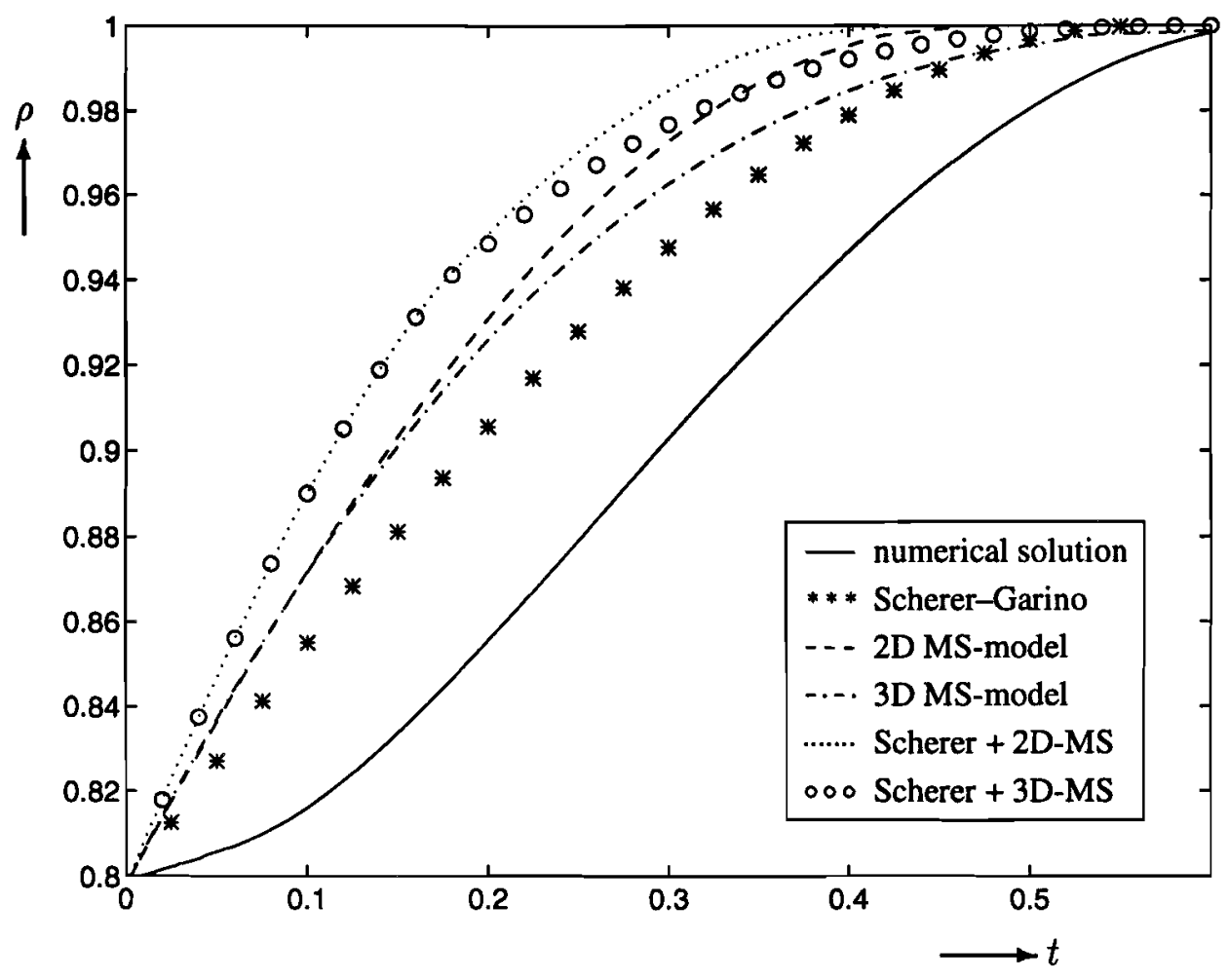

Figure 17: During the early stage the numerically obtained densification rate differs quite a lot with the prediction of the analytic shrinkage models.

of cylindrical particle packings.

The simplest densification problem in this class is the sintering of a regular packing of equally sized cylinders. A part of this packing is shown in figure 16. The initial radii of all the cylinders is taken equal to 0.5 and the contact radius, say $r$, between two touching cylinders is initially set equal to 0.095 for all neck regions. Furthermore, we use Hopper's analytical solution for the coalescence of two equal cylinders to approximate the neck regions of the initial shape (cf. Hopper [12]). In figure 16 we show the densification of the unit cell at equal periods of 0.1 . As can be observed from these pictures, the pore shape evolves towards a smooth curved geometry of the type we considered in the previous subsection, viz. the square pore with rounded-off corners of figure 7.

In figure 17 we have compared the densification rate of the cylindrical packing geometry to the various analytical models. Now we observe that these relations predict a quite different densification evolution during the early stage compared to the numerical simulation results: the numerically obtained rate proceeds much slower than the analytical predictions. This is caused by a smoothing of the neck region during this stage, which results in only a small reduction of the pore size. Hence this example illustrates again that the densification rate depends on the pore shape of the initial geometry.

An interesting question is the behavior of the neck growth between two touching cylinders of the packing compared to the exact contact radius development of two equal coalescing cylinders, 


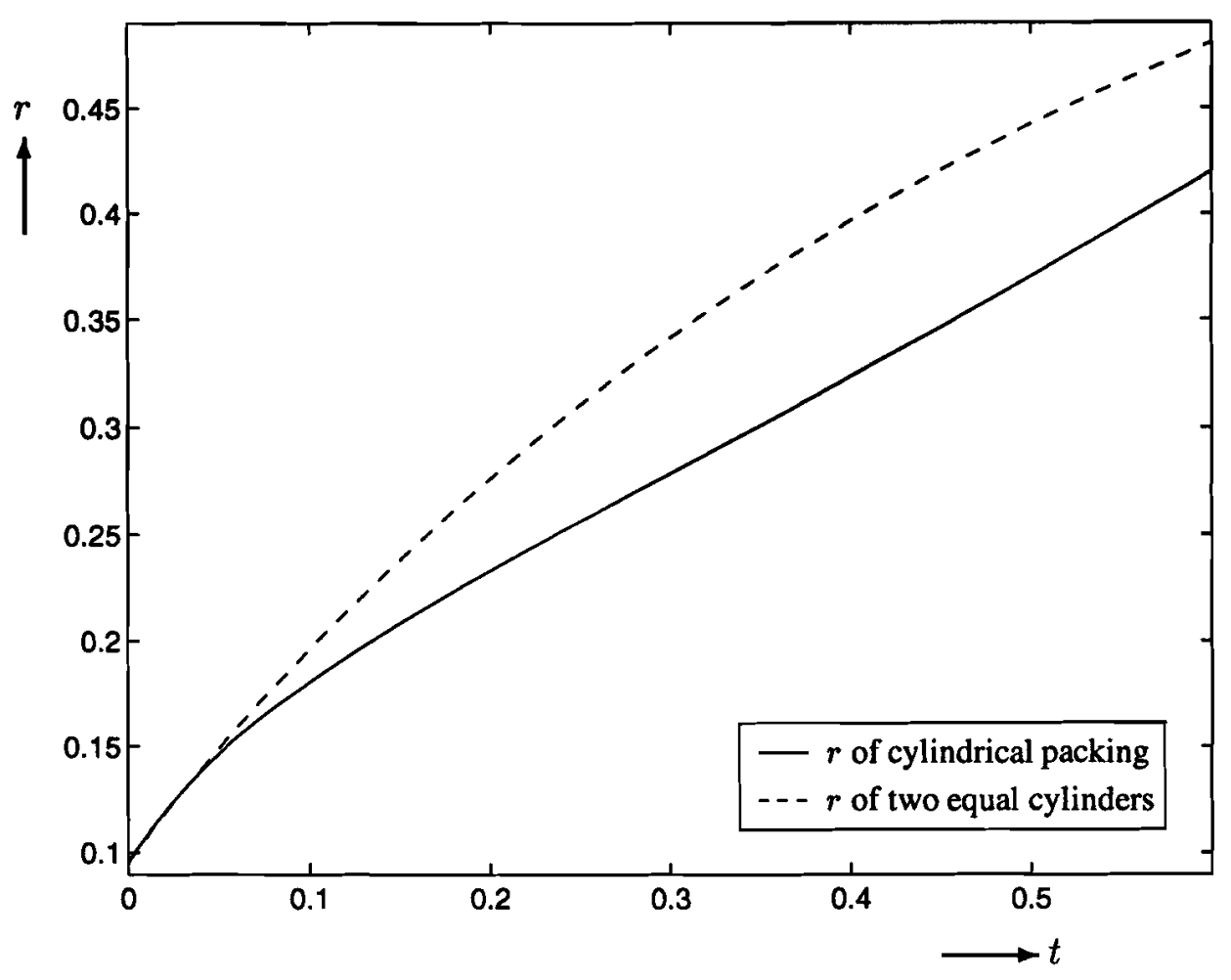

Figure 18: The contact radius $r$ development of the cylindrical packing compared with the exact analytical solution of the coalescence of two equal cylinders shows only an agreement during the early stage. At later time stages the neck radius of the cylindrical packing develops much slower.

i.e. the time stage for which the analytical solution is applicable. In figure 18 we show the development in time of the contact radius for the unit cell. We also plotted the exact neck radius for the coalescence of two cylinders with initial radii equal to 0.5 , as obtained from Hopper's analytical solution. From this figure we observe that only during the initial stage the neck growth is similar to the contact radius development of two coalescing cylinders, i.e. during the smoothening of the neck region when $t<0.08$. Afterwards, the contact radius increases much slower compared with the analytic solution. Hence the latter prediction should only be used during the early stage of the sintering process.

Next, we investigate the effect of the cylindrical particles on the densification rate when those particles are the building blocks of the pore structure. The simplest model for such a gel network is obtained by taking a square unit cell with one pore and each boundary edge consisting of a straight line of initially coalesced cylinders. Hence this cell might be seen as a sophisticated update of the rounded off square pore shape that we considered in subsection 3.1.

The model above is also of interest for the sintering of base-catalysed aerogels, since the microstructure of such an aerogel can be described by the following model (cf. Emmerling et al. [30]): small amorphous particles with diameter $1 \mathrm{~nm}$ form the building blocks of so-called secondary particles with a diameter of approximately $5 \mathrm{~nm}$ (depending on the $\mathrm{pH}$ value of the sol-gel process); these secondary particles form the branched gel network which consists of open 

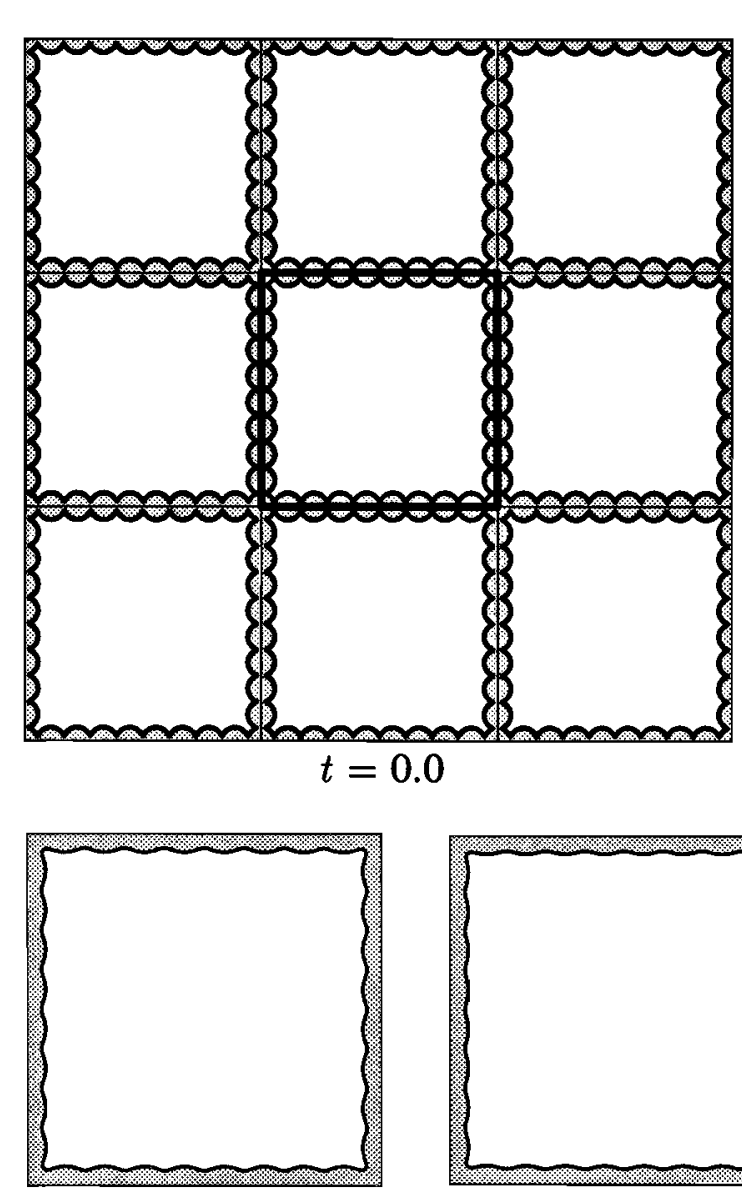

$t=0.4$

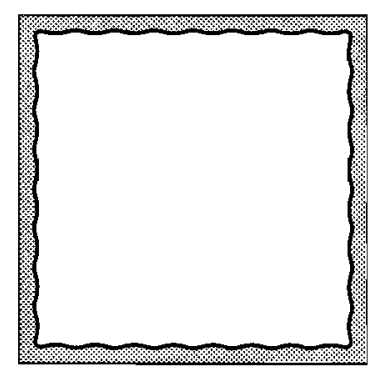

$t=0.5$
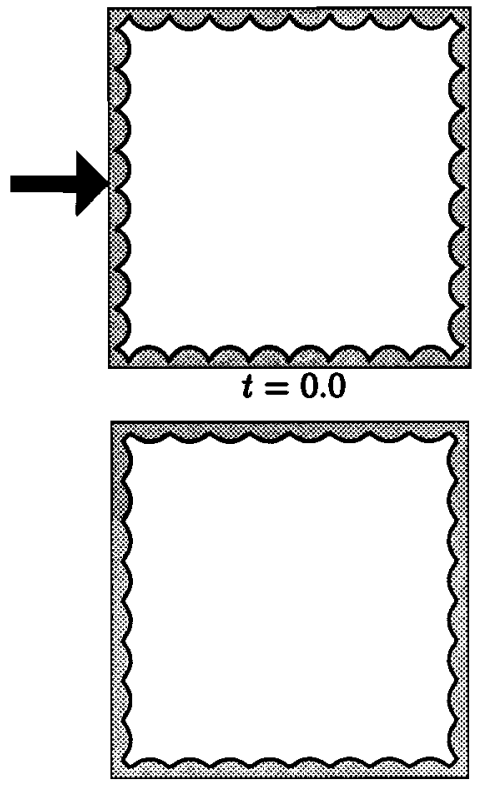

$t=0.2$

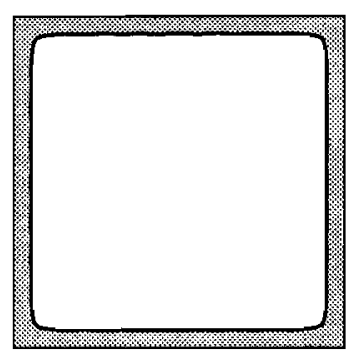

$t=1.0$
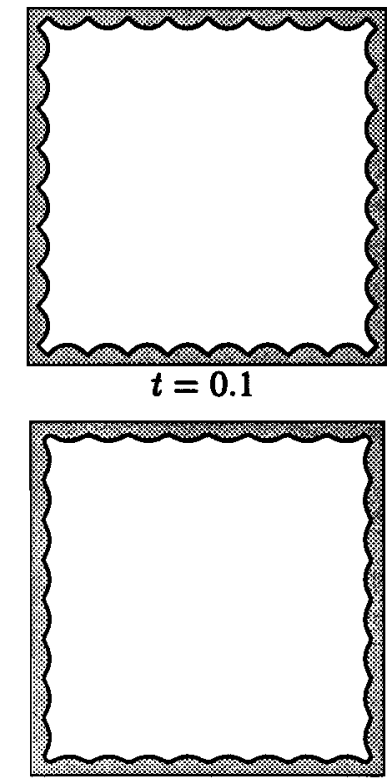

$t=0.3$

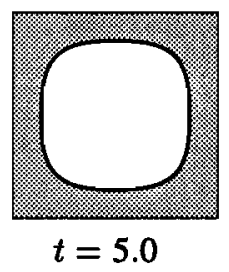

Figure 19: The shape deformation of a periodic square unit cell whereby each edge consists of 10 equal coalesced cylinders shows that during the early stage the cell expands before it shrinks towards a rounded-off square pore shape like the one we considered in figure 6.

macropores with a mean diameter of about $50 \mathrm{~nm}$ (depending on the macroscopic density of the aerogel). In the texture model of the base-catalysed aerogel that we considered in the previous subsection (see figure 14), both the primary and secondary particles were assumed to be coalesced completely. This is also expressed in the (relatively) high initial density of that model. In order to investigate the effects due to the coalescence of the secondary particles, we assume that all those particles are of equal size and of cylindrical form. Hence the primary particles are ignored and are assumed to be completely coalesced to these cylindrical particles.

Here we consider this type of unit cell with $m$ cylindrical particles along each edge, where $m=2,4, \ldots, 12$. The neck radius between two successive cylinders is taken initially equal to 0.175 and the neck shape is obtained from Hopper's analytical solution. In figure 19 we have plotted a typical shape deformation of such a model whereby the unit cell consists of 10 by 10 cylindrical particles of equal radii of 0.5 . From the figure, we observe that during the coalescence of the cylindrical particles the cell shrinks like a rounded-off square pore shape. Hence we may 


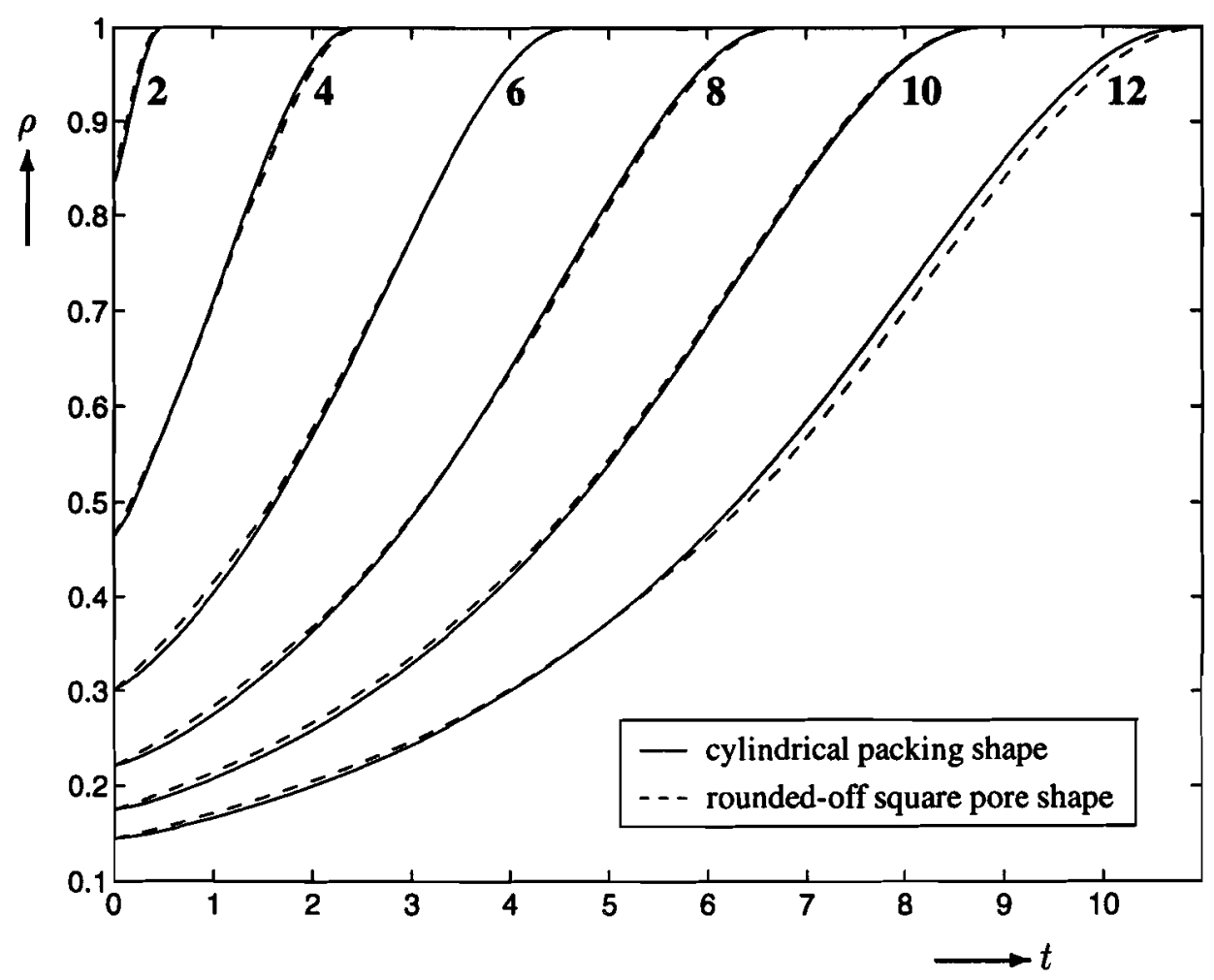

Figure 20: The solid lines denote the density $(\rho)$ change of the cell with initially $m$ cylindrical particles along each edge, where $m=2,4, \ldots, 12$. The dashed lines show the densification behavior of the initially rounded-off square pore shapes with corresponding initial density.

expect a good agreement between the densification of the above unit cell and the rounded-off pore shape which is demonstrated in figure 20. In that figure we have plotted the densification development of the 6 considered unit cells by solid lines. Moreover, the densification rate of the rounded-off pore shapes with the same initial density are also plotted by dashed lines. From the close matching of the corresponding unit cells we can conclude that modelling the aerogel by a network of cylindrical particles does not give new insights in the densification kinetics of the gel. However, from a computational point of view the simulation of the rounded-off pore shapes is much easier and less CPU-time consuming than the particle network.

\section{Conclusion}

The examples of section 3 demonstrate the capabilities of our numerical approach in obtaining theoretical insights with respect to both pore size and pore distribution of the sintering gel. In particular the model is able to examine the microstructure evolution during the densification of a particular pore size distribution.

The major findings are that pores seem to vanish in order of size: first the smallest pores followed subsequently by the larger ones. Due to this vanishing order there is not a 1-1 correspondence between the initial total free surface and the densification end time as is illustrated 
by figure 13. Another interesting phenomenon is the observation that some pores may grow during a certain time when those pores have concave boundary parts, i.e. such a pore has a much longer boundary length than it would strictly require to surround the pore contents. Hence, due to the "excess" of boundary, the pore may expand whereas the total pore boundary length still decreases as time evolves.

It is also shown that the Scherer-Garino equation (3.4) usually provides the most accurate approximation of the numerically obtained densification results, regardless of the pore shape and distribution. Since this equation is especially developed to analyze the sintering of a porous glass layer on a rigid substrate, the presented two-dimensional simulation code should be applicable to simulate the sintering of such coatings. However, some experimental verification has to be carried out to justify the latter statement. In the near future we also hope to say something about the applicability of the proposed mathematical approach in the case of sintering aerogels (cf. Emmerling et al. [31]). In that paper experimental data will be validated using the rounded-off square pore unit cell of subsection 3.1.

It is the author's opinion that it is also possible to introduce the occurrence of fixed particles in the mathematical model; this in order to simulate two phase viscous sintering. Moreover, if really three-dimensional numerical viscous sintering simulations are required, a similar approach should be followed as proposed in in this paper. In principle, only the governing integral equation would be different. However, taking in consideration the required CPU time for the two-dimensional calculations, the limits in computer resources will soon be reached when trying to simulate the sintering of a more complicated unit cell.

\section{Acknowledgement}

The author would like to express his appreciation to Prof. G. de With of Philips Research Laboratory for the stimulating discussions, Dr. G.W. Scherer of DuPont de Nemours \& Co. and Prof. C. Pozrikidis of the University of California at San Diego for giving useful comments. Prof. R.M.M. Mattheij of Eindhoven University of Technology is thanked for providing the facilities to finish this work and for critically reading the manuscript.

\section{References}

[1] C.J. BRINKER AND G.W. SCHERER, Sol-gel science : the physics and chemistry of sol-gel processing. Academic Press, London, 1990.

[2] H.K. KUIKEN, Viscous Sintering: the Surface-Tension-Driven Flow of a Liquid Form under the Influence of Curvature Gradients at its Surface, J. Fluid Mech. 214, 503-515, 1990.

[3] J. FRENKEL, Viscous Flow of Crystalline Bodies Under the Action of Surface Tension, $J$. Phys. USSR 9, 385-391, 1945.

[4] G.A.L. VAN DE VORST, Numerical Simulation of Axisymmetric Viscous Sintering, Eng. Analysis with Boundary Elements, 14(2), 193-207, 1994. 
[5] W.D. KINGERY AND M. BERG, Study of the Initial Stages of Sintering by Viscous Flow, Evaporation-Condensation, and Self-Diffusion, J. Appl. Phys. 26, 1205-1212, 1955.

[6] H.E. EXNER AND G. PETzOw, Shrinkage and Rearrangement During Sintering of Glass Spheres, in Proc. Conf. on Material Science Research, Vol. 10 Sintering and Catalysis, edited by G.C. Kuczynski, Plenum Press, New York, pp. 279-293, 1975.

[7] J.K. MACKenZiE AND R. ShutTleworth, A Phenomenological Theory of Sintering, Proc. Phys. Soc. Lond. 62, 833-852, 1949.

[8] G.W. SchERER, Sintering of Low-Density Glasses: I, Theory, J. Am. Ceram. Soc. 60, 236-239, 1977.

[9] G.W. SCHERER, Viscous Sintering of a Bimodal Pore-Size Distribution, J. Am. Ceram. Soc. 67, 709-715, 1984.

[10] G.W. Scherer AND T. GaRINO, Viscous Sintering on a Rigid Substrate, J. Am. Ceram. Soc. 68, 216-220, 1985.

[11] R.W. HOPPER, Coalescence of two equal cylinders - Exact results for creeping viscous plane flow driven by capillarity, J. Am. Ceram. Soc. 67, 262-264, 1984.

[12] R.W. HoPPER, Plane Stokes Flow Driven by Capillarity on a Free Surface, J. Fluid Mech. 213, 349-375, 1990.

[13] R.W. HOPPER, Plane Stokes Flow Driven by Capillarity on a Free Surface, 2: Further Developments, J. Fluid Mech. 230, 355-364, 1991.

[14] R.W. HOPPER, Stokes Flow of a Cylinder and Half-Space Driven by Capillarity, J. Fluid Mech. 243, 171-181, 1992.

[15] S. RICHARDSON, Two-dimensional slow viscous flows with time-dependent free boundaries driven by surface tension, Euro. J. App. Math. 3, 193-207, 1992.

[16] J.W. Ross, W.A. Miller AND G.C. WEATHERLY, Dynamic Computer Simulation of Viscous Flow Sintering Kinetics, J. Appl. Phys. 52, 3884-3888, 1981.

[17] A. Jagota AND P.R. DAwson, Micromechanical Modeling of Powder Compacts-I. Unit Problems for Sintering and Traction-Induced Deformation, Acta. Metall. 36, 2551-2561, 1988.

[18] A. JAgOTA AND P.R. Dawson, Micromechanical Modeling of Powder Compacts-II. Truss Formulation of Discrete Packings, Acta. Metall. 36, 2563-2573, 1988.

[19] A. JaGota AND P.R. DAwson, Simulation of the Viscous Sintering of Two Particles, J. Am. Ceram. Soc. 73, 173-177, 1990. 
[20] A. JAGota, Simulation of the Viscous Sintering of Coated Particles, J. Am. Ceram. Soc., 1995. (To appear).

[21] G.A.L. VAN DE VORST, R.M.M. MATTHEIJ AND H.K. KUIKEN, Boundary Element Solution for Two-Dimensional Viscous Sintering, J. Comput. Phys. 100, 50-63, 1992.

[22] G.A.L. VAN DE VORST AND R.M.M. MATTHEIJ, Numerical Analysis of a 2-D Viscous Sintering Problem with Non Smooth Boundaries, Computing 49, 239-263, 1992.

[23] G.A.L. VAN DE VORST, Integral Method for a Two-Dimensional Stokes Flow with Shrinking Holes Applied to Viscous Sintering, J. Fluid Mech. 257, 667-689, 1993.

[24] G.A.L. VAN DE VORST, Modelling and Numerical Simulation of Viscous Sintering, PhD thesis, Eindhoven University of Technology, 1994.

[25] G.A.L. VAN DE VORST AND R.M.M. MATTHEIJ, A BEM-BDF Scheme for Curvature Driven Moving Stokes Flows, J. Comput. Phys., 1995. (To appear).

[26] G.A.L. VAN DE VORST, Integral Formulation to Simulate the Viscous Sintering of a TwoDimensional Lattice of Periodic Unit Cells, J. Eng. Math., 1995. (To appear).

[27] G. DE WITH, Meso-modelling: a proposed attempt to obtain an industrially applicable sintering theory, in Proc. Conf. on Modelling of sintering processes, 1990. Lecture at the 71 WE Heraeus Seminar.

[28] G.K. BATCHELor, An Introduction to Fluid Dynamics, Cambridge University Press, Cambridge, 1967.

[29] A. Craievich, M.A. Aegerter, D.I. dos SANTos, T. Woignier and J. ZarzyCKI, A SAXS Study of Silica Aerogels, J. Non-Cryst. Solids 86, 394-406, 1986.

[30] A. Emmerling, R. Gerlag, R. Goswin, J. Gross, G. Reichenauer, J. Fricke AND H.G. HAUBOLD, Structural Modifications of Highly Porous Silica Aerogels upon Densification, J. Appl. Cryst., 24, 781-787, 1991.

[31] A. EMmerling, W. Lenhard, J. Fricke AND G.A.L. VAN DE VorST, Densification Behaviour Aerogels upon Isothermal Sintering, 1995. (In preparation). 